\title{
Complete Body Aerodynamic Study of three Vehicles
}

\author{
Simmonds N, Pitman J, Tsoutsanis P, Jenkins K, Gaylard A, and Jansen W.
}

\begin{abstract}
Cooling drag, typically known as the difference in drag coefficient between open and closed cooling configurations, has traditionally proven to be a difficult flow phenomenon to predict using computational fluid dynamics. It was seen as an academic yardstick before the advent of grille shutter systems. However, their introduction has increased the need to accurately predict the drag of a vehicle in a variety of different cooling configurations during vehicle development. This currently represents one of the greatest predictive challenges to the automotive industry due to being the net effect of many flow field changes around the vehicle.
\end{abstract}

A comprehensive study is presented in the paper to discuss the notion of defining cooling drag as a number and to explore its effect on three automotive models with different cooling drag deltas using the commercial CFD solvers; STARCCM+ and Exa PowerFLOW. The notchback DrivAer model with under-hood cooling provides a popular academic benchmark alongside two fully-engineered production cars; a large saloon (Jaguar XJ) and an SUV (Land Rover Range Rover). Initially three levels of spatial discretization were used with three steady-state RANS solvers (k- $\varepsilon$ realizable, k- $\omega$ SST and Spalart-Allmaras) to ascertain whether previous work using RANS on the large saloon studying cooling flows could be replicated on other vehicle shapes.

For both the full-production vehicles, all three turbulence models were capable of predicting the cooling drag delta within 5 counts $\left(0.005 C_{d}\right)$. However, the DrivAer model was much more sensitive to both changes in turbulence models and mesh sizes. For the SA turbulence model only the drag coefficient was well predicted, for the other two RANS models no amount of grid refinement allowed the models to correctly predict the flow field. It was seen when comparing the $\mathrm{k}-\varepsilon$ realizable and SA turbulence models the difference in cooling drag was attributed to the rear of the vehicle. This highlighted that despite similar drag values from the cooling package, the cooling deltas were very different, suggesting that cooling drag cannot be thought of as open-closed drag with the addition of drag due to the cooling package.

Further work on the DrivAer model expanded on the RANS simulations utilizing the eddy-resolving methods, IDDES and LBM, as validation cases. Oscillations which were seen in the SA and k- $\omega$ SST RANS turbulence models were shown to be of similar levels to those in the transient methods indicating a pseudo-unsteadiness present in the steady-state solvers and the importance of resolving it. Drag and lift coefficient absolute values were compared showing that only the IDDES method with sliding wheels and LBM method could obtain physical results for the majority of the tested criteria.

\section{Introduction}

Current and future regulations in the automotive industry place a high importance on the environmental impact of vehicles. It is becoming increasingly important to be able to calculate the drag of each vehicle specification and the affect of each changeable component on the final configuration. Wind tunnels can obtain all this information but it is difficult to create representative prototypes early enough in the development process. With ever increasing computational power available Computational Fluid Dynamics (CFD) provides the ability to simulate and calculate the drag configurations for a variety of models, while state-of-the-art multi-physics simulations with coupling of aerodynamics, thermal management and power-train simulations provide the potential to model real-time simulations.

This paper focuses on increasing understanding of so-called cooling air drag, as applied to a fully engineered production large saloon and SUV, this is achieved by discussing the concept of cooling drag vs. complete body aerodynamic simulations and comparing against the popular benchmark DrivAer model.

\section{Cooling Air Flows}

Providing a cooling mechanism for a typical automotive internal combustion engine has traditionally involved passing a coolant around the engine block to absorb residual heat, this hot liquid is channeled to a heat exchanger(s) through a series of pipes where it transfers its heat to fins and finally via convection to the ambient air passing through the heat exchanger. This process requires a constant flow of air through the heat exchanger, which is provided by openings on the front of the car in areas of high stagnation pressure.

The introduction of high velocity flow through the engine bay has been reported to dramatically increase the drag of the vehicle by up to $20\left(0.02 C_{d}\right)$ and $30\left(0.03 C_{d}\right)$ counts for Saloon and SUV type models. This difference in drag has become known as the "cooling drag" of a model and has been obtained experimentally by closing the intake grilles.

Current cooling air flow CFD studies can be traced to work undertaken on generic models such as the Ahmed body, modified with an internal duct, as performed by Barnard [1,2]. More recent work in the field has taken advantage of modern computational resources in modeling full vehicle airflow problems on production

Page 1 of 19 


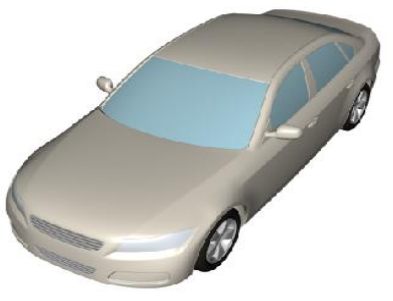

(a) DrivAer

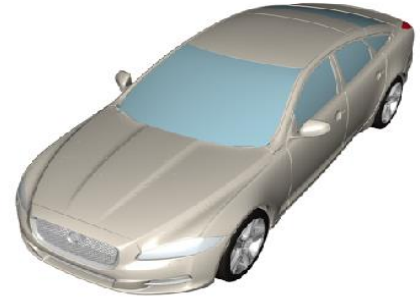

(b) Large Saloon

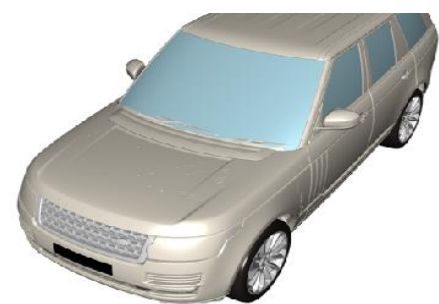

(c) SUV

Figure 1. Three automotive models to be tested: The DrivAer Model, a large production saloon and a production SUV.

automotive geometries such as Large Saloons $[3,4,5]$ and SUVs [3, $6,7,8,9]$.

The complex flow interactions behind the introduction of cooling air flow has proven an obstacle in obtaining an accurate prediction using CFD. These interferences have been an important field of study in cooling airflows and have been well documented. [10, 11, 12, 13]. Other relevant work in this subject has focused on studying the effect of various components on the system $[5,9,14]$.

Most recently the DrivAer model was seen as an ideal candidate to modify by including underhood geometry, in order to provide a benchmark case which could be introduced for validation [12]. This has allowed for geometrical changes and for external factors such as wind tunnel affects to be modeled $[13,15]$.

\section{Cooling-Drag Assessment}

The cooling drag coefficient is a measure of the drag difference between and open and closed grille vehicles. It is a widely used and accepted measure in the automotive aerodynamics community for characterizing cooling flows. The coefficient itself has many issues which have been documented with the major problems originating from the change of flow physics due to closing the grilles and the affect this has on other components downstream. The coefficient is typically written as seen in Equation 1, in order to obtain a positive value. An efficient cooling system is a pre-requisite for a low 'cooling drag' value however the latter is not a direct indicator of the former. The few cases with apparently negative 'cooling drag' give an indication that this quantity does not simply represent the momentum loss through the cooling pack and engine bay and that the term is somewhat misleading.

$$
C_{D_{\text {cooling drag }}}=C_{D_{\text {with cooling flow }}}-C_{D_{\text {without cooling flow }}}
$$

\section{Research Aims}

There are three main motivations of this paper. Initially the paper explores the notion of measuring cooling drag as a number, with a shift in emphasis towards choosing the correct CFD method and settings to correctly capture the aerodynamics of a vehicle regardless of whether the grills are open or closed, or the type of geometry present. The paper aims to build on established literature for the DrivAer model where DES simulations have been performed without the underhood regions or more complex boundary conditions (such as moving ground and wheels) $[16,17]$. Finally, the paper explores the Page 2 of 19 connection between the DrivAer case and full production road vehicles.

\section{Automotive Models}

A comprehensive study is presented in this paper of three automotive models, seen in Figure 1, with different reported cooling drag deltas. The notchback DrivAer model with under-hood cooling provides a popular academic benchmark alongside two fully-engineered production cars; a large saloon (Jaguar XJ) and an SUV (Land Rover Range Rover). Due to the wealth of available data on the DrivAer model this will be presented first in order to validate the simulation set-up and act as a reference for future publications. The two production cars have the following experimentally reported cooling drag deltas:

- $\quad$ Large Saloon $=25$ Counts $\left(0.025 \mathrm{C}_{\mathrm{d}}\right)$

- $\mathrm{SUV}=35$ Counts $\left(0.035 \mathrm{C}_{\mathrm{d}}\right)$

The DrivAer model is available to download from the Technical University of Munich (TUM), first introduced in 2012 [18] it has become the standard for validation in automotive cases using a generic model. The geometry is based on two medium saloons, the BMW 3 series and the Audi A4, and was created by merging simplified CAD models in a collaboration between the BMW Group, Audi AG and the Institute of Aerodynamics and the Fluid Mechanics group of the Technische Universitat Munchen (TUM).

The geometry provides a variety of options:

- Open and Closed Wheels

- Detailed or Smooth Underbody

- Three rear-end configurations (fastback, notchback and estate)

This geometry was modified in 2015 [12], where an engine bay compartment was added. In addition to the original components the new geometry contains a body with open front grilles, an underbody with engine bay outlet, a cooler, a radiator (to be modeled as a porous media), an engine, a gearbox and an exhaust system.

The model is designed for further study by providing two variants of the cooler. One without any leakage, which has been previously published $[12,13]$ and one which contains leakage around the cooling package.

During this paper the Notchback model will be studied with open rotating wheels, front grilles, mirrors and the cooler without leakage, 


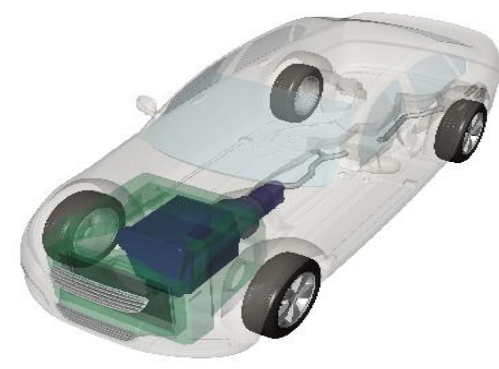

(a.) DrivAer

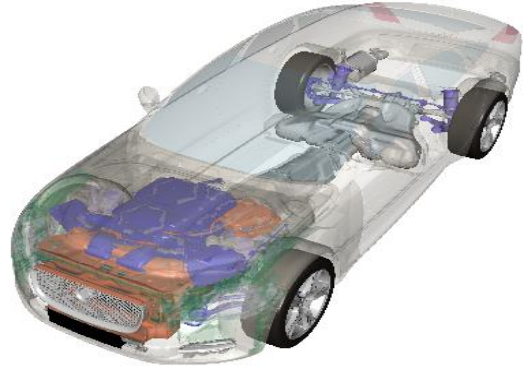

(b) Large Saloon

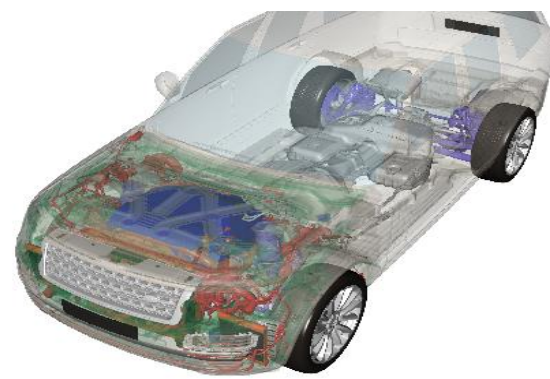

(c) SUV

Figure 2. Detailed underhood geometry of the three modelled vehicles, outlining the added complexity of the full production vehicles vs. the DrivAer model.

this is to match the conditions of previous cooling work [13]. While not expanded upon during this paper it has been found that the leakage cooler configuration can increase the cooling drag coefficient by at least 10 counts $(0.01 \mathrm{Cd})$.

The closed grille case was modelled by simply blanking the inlet apertures of the 'open cooling' case.

It should be recognised that the model in the current paper has a slightly different set-up to the previous studies, to which it will be compared. A few modelling decisions related to the following points for the DrivAer are described below:

- Contact patches or tangential tyres

- Wheel surface roughness

- Scale

- 5-belt system

- Wind tunnel boundary conditions

The tyres are modelled tangential to the ground, instead of typically used contact patches, to match the experimental use of metallic wheels. Similarly, the use of metallic wheels led to the decision to use zero surface roughness. However, these simulations do not attempt to model other wind tunnel boundary conditions (struts etc.) or the 5 belt system used in the experiment, these simulations can be thought of as "open road", they are also run at full scale with a corresponding car length (L) of $4.6 \mathrm{~m}$. The DrivAer model was kept at full scale in order that the findings of the study be more directly comparable with the production vehicles.
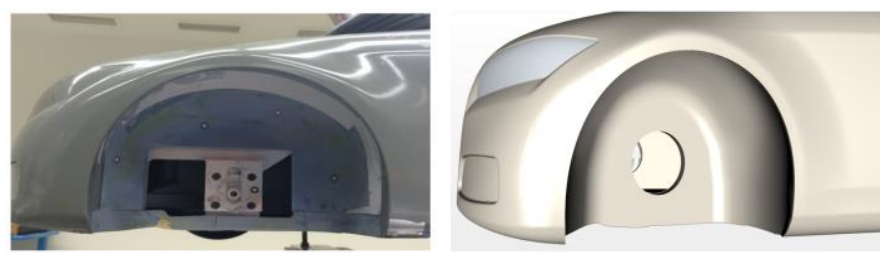

Figure 3. Wheel arch openings showing difference between experimental square baseline [13] and CAD geometry circular opening

The final difference in these simulations compared to the previous work is the wheel arches as seen in Figure 3. The baseline experimental wheel arch is square and larger than that available to download from the TUM archive, the large experimental wheel apertures result in 3/4 of the engine bay flow exiting through the Page 3 of 19 wheel arches [12]. It has been confirmed to the authors that the CFD simulations in the previous work also used the larger square wheel arches. Therefore, this will be an area of uncertainty with respect to comparison to the previous simulations but satisfies a motivation to create a fully reproducible simulation for future validation.

Figure 2 details the underhood components involved in the simulations. The simiplicity and scarcity of the DrivAer model components in the engine bay can be seen in comparision to the Large Saloon and SUV models.

\section{Numerical Methods}

The numerical simulations presented in this paper were performed primarily using the commercial CFD solver STARCCM+ v11.02. For each vehicle three Reynolds Averaged Navier-Stokes (RANS) turbulence models were used, while an Improved Delayed Detached Eddy Simulation (IDDES) was also performed for the DrivAer model. For additional comparison, Exa PowerFLOW was also utilized. The Lattice Boltzmann Solver (LBM) was used successfully to perform the computational component of previous work [13]. In the present work it will be used to validate differences in the results that may arise from the differences in the simulation set-up (wheel arches, wind tunnel effects and scale).

\section{Starccm+}

STARCCM+'s segregated flow model was enabled, for both RANS and IDDES simulations, which solves the discretized flow equations in an uncoupled manner using a predictor-corrector (SIMPLE algorithm) approach to link the momentum and continuity equations. It is second order accurate for the convective terms and uses a GreenGauss gradient reconstruction for improved stability.

All STARCCM+ simulations were undertaken using Cranfield University's Astral High Performance Computer (HPC) cluster on 256 Intel cores (Intel E5-2660) with a minimum of 4GB ram per core.

\section{RANS}

The three turbulence models included in this study are SpalartAllmaras (SA) [19], $\mathrm{k}-\varepsilon$ realizable [20] and $\mathrm{k}-\omega$ Shear Stress Transport (SST) [21]. All models were solved to convergence with default settings for turbulence parameters and velocity and pressure 
under-relaxation factors. All models converged between 4000-10000 iterations, dependent on vehicle and mesh resolution. Fluctuations were noticed in the flow fields for both the SA and $k-\omega$ SST flow fields. Flow averaging was performed on these models once stable for 5000 iterations to obtain mean velocity and pressure fields. Total simulation time on Cranfield University's Astral HPC was in the range of 0-5 days.

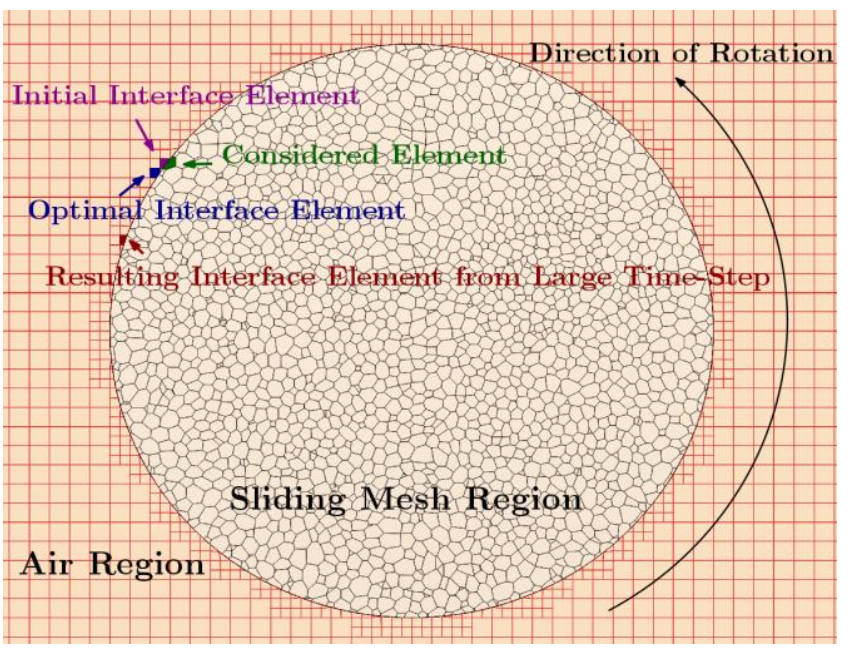

Figure 4. Fluid and sliding mesh regions for the IDDES solver showing the effect of a large time-step on wheel rotation.

\section{IDDES}

The original Detached Eddy Simulation (DES) was constructed with the SA RANS turbulent model as a hybrid between unsteady RANS and Large Eddy Simulations (LES). LES relies on very fine isotropic cells in the boundary layer. By utilizing URANS in this region DES greatly improves computational time against LES. IDDES is an improvement to traditional DES approaches typically reducing errors in the transition between the RANS models employed in the boundary layer and, if the grid is fine enough, LES sub-grid scale model.

The IDDES simulations performed on the DrivAer model in STARCCM+ were based upon the $\mathrm{k}-\omega$ SST RANS turbulence model. The unsteady simulation was performed on the medium meshes for the DrivAer with a time step of $\Delta T=3.5 \times 10^{-4}$. This resulted in CFL values of 1 or less throughout the domain. While it would be possible to use larger time-steps on a coarser mesh, the presence of rotating wheels complicates the simulation. In order to achieve an optimal IDDES set-up with rotating wheels, it is suggested that the mesh rotates one element of the air region per time-step, as seen in Figure 4. If a larger time-step is used to take advantage of a coarser mesh, the considered element of the sliding mesh region would interface with an element too far round the rotation, reducing the accuracy of the solution and potentially causing it to crash. The finest mesh was not used for the IDDES runs due to its computational expense. The proposed time-step for the medium mesh was previously shown to produce accurate drag values for the full scale DrivAer without the engine bay compartment on a very coarse DES mesh [22]. Previous work has also shown the IDDES solvers ability to perform well on coarser meshes, further validating the decision not to utilize the finest mesh.

The simulations were initialized from the $\mathrm{k}-\omega$ SST RANS simulations followed by a period of 5 flow passes ( 1 second real Page 4 of 19 time) to enable the solution to stabilize. In order to obtain timeaveraged statistics the flow was progressed for a further 10 passes $(2$ seconds real time). Time was discretized using a 2 nd order method.

\section{PowerFLOW}

Version 5.1b of EXA PowerFLOW was used for this study. PowerFLOW is an extended Lattice Boltzmann solver which simulates discrete fluid particles, represented by particle densities in cells called 'voxels' which move in discrete space and time.

The cartesian grid is intersected by the geometry, at which locations the voxels are 'cut', to form a surface element, or 'surfel'.

Momentum from the fluid is transferred to the surface via collision equations. This has several key advantages in the industrial environment - one being that the input faceted geometry can have high aspect ratio elements and the other that the discretization process is fully automated with no input from the user. Refinement regions are specified at the case set-up stage in areas of strong pressure gradient, small gaps or separation locations. The methodology is termed Very Large Eddy Simulation (VLES) and for high Reynolds number applications uses a proprietary version of the k- $\varepsilon$ RNG subgrid turbulence model along with an extended wall model in boundary layer regions.

Simulations are run until a set of proprietary stability criteria for the drag and lift forces are met for a set period of flow-passes. An initial transient settling phase of the simulation is determined and removed before time averaging. The length of the simulation varies between vehicle types and can be on the order of 5-6 seconds of physical time for vehicles with low frequency wake oscillation. For the DrivAer model the physical time for the averaged region is on the order of 1-2 seconds. Mesh refinement around the vehicle was specified based on the EXA best practice guidelines for automotive applications, with a finest cell size of $1.25 \mathrm{~mm}$. With a flow velocity of $100 \mathrm{~km} / \mathrm{hr}$, the simulation time-step is on the order of $10^{6}$ seconds. Wheel rotation is realised with sliding mesh regions for the wheel spokes and rotating wall boundary conditions on the rest of the axisymmetric geometry of the wheel.

All PowerFLOW simulations were run on the JLR HPC facility, using 192 CPUs. Simulations performed in PowerFLOW will henceforth be referred to as the Lattice Boltzmann Method (LBM).

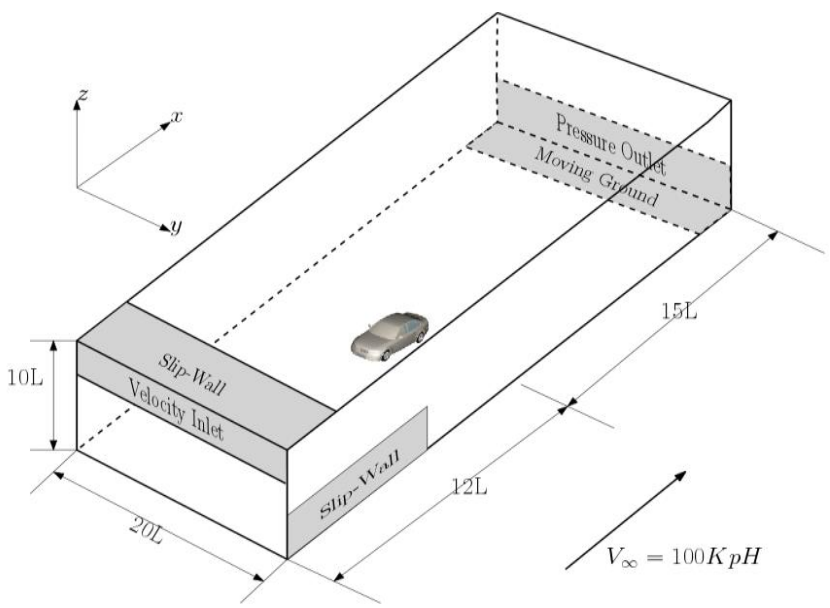

Figure 5 Computational Domain and Boundary Conditions. 


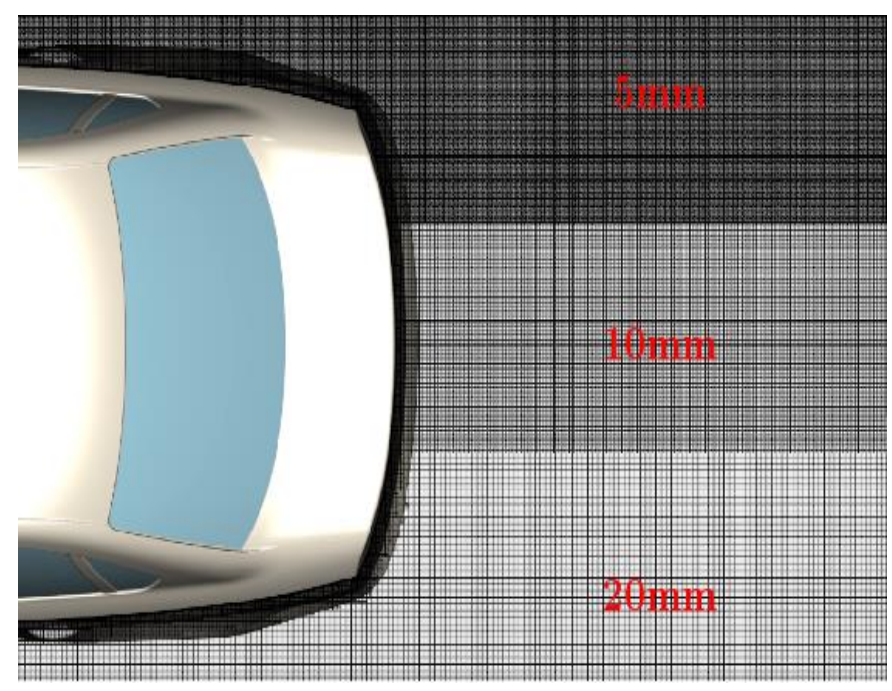

Figure 6. Wake resolution for three different meshes to be tested showing the effect of cell edge length.

\section{Computational Set-Up}

\section{Boundary Conditions}

For the STARCCM+ simulations, the domain is set-up in an "openroad" configuration with a velocity inlet located $12 \mathrm{~L}$ upstream of the vehicle and a pressure outlet $15 \mathrm{~L}$ downstream. The top and sides of the domain are defined as adiabatic slip wall boundaries and are at least $10 \mathrm{~L}$ from the vehicle. The computational domain can be seen in Figure 5. These domain settings in STARCCM+ have previously resulted in accurate predictions for the cooling drag coefficient [5], the domain will be used for all models whereby they will be switched out and the center of the front of the vehicles positioned at $(0.5,0,0)$ $\mathrm{m}$.

The freestream for all simulations is kept constant at $100 \mathrm{KpH}$ with a density of $1.184 \mathrm{Kg} / \mathrm{m}^{3}$. Rotating wheels are modeled by Moving Reference Frames (MRF) in RANS and one configuration of the IDDES simulations to bridge the gap between the steady-state and transient simulations. A detailed discussion on the implementation of MRF's in this set-up can be seen in previous work by Simmonds et al [5]. Sliding mesh approaches were used for both IDDES and LBM methodologies.

The radiators and condensers for all three vehicles are modeled using porous media. Seen in previous work by Simmonds et al [5] and by Kuthada et al [13] pressure drop changes over a radiator have very small effects on the cooling drag. The DrivAer pressure drop values used in this paper in order to be neutral and easily reproducible are as follows: inertial resistance $=100 \frac{\mathrm{kg}}{\mathrm{m}^{4}}$ and the viscous resistance $=$ $700 \frac{\mathrm{kg}}{\mathrm{m}^{3} \mathrm{~s}}$.

\section{Grid Resolution}

During this study the effect of grid resolution was tested in order to obtain the most accurate solution. All three vehicles underwent surface wrapping in STARCCM+ to efficiently resolve the detailed engine bay components. The original aero surfaces, those typically exposed to freestream velocities, were manually meshed from CAD and then re-sewn into the geometry to remove any inaccuracies. The volume meshes in STARCCM+ were constructed using the trimmed cell and prism layer meshers. The prism layer was developed from an initial wall cell height corresponding to a y+ value in the 30-100 range, this allowed for an accurate deployment of the all $\mathrm{y}+$ wall function. The prism layers combined for a total wall normal height of $5 \mathrm{~mm}$ to keep the total cell count down. A small prism layer was successfully utilized by Forbes et al using the k- $\omega$ SST IDDES in Star CCM+ [22].

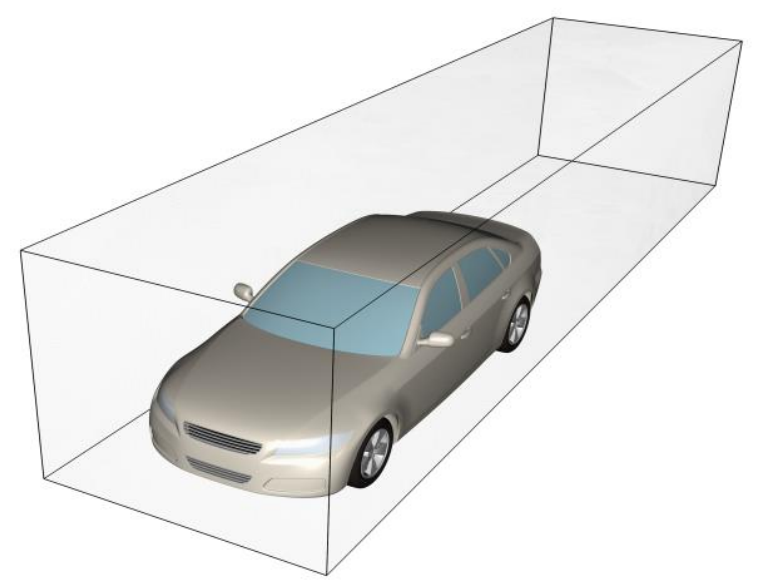

Figure 7. Density Box outlining region of refinement for cells closest to model.

The simulation matrix undertaken in this study tested three RANS turbulence models on three grid resolutions, seen in Figure 6. For all meshes the prism layer mesher was kept constant, while the density box seen in Figure 7, defining maximum cell size contained within it, closest to the vehicle was changed. The density box's cell size was based upon its edge length; tested were $20 \mathrm{~mm}, 10 \mathrm{~mm}$ and $5 \mathrm{~mm}$ lengths. The corresponding total volume mesh sizes for the corresponding edge length and vehicle can be seen in Table 1. The large increase in element count for the $5 \mathrm{~mm}$ meshes results from halving the edge length, therefore splitting the original element into 8 .

Table 1: Grid resolution used in study for three models based on the smallest wake elements.

\begin{tabular}{cccc}
\hline Model & Refinement & $\begin{array}{c}\text { Wake Element } \\
\text { Edge Length } \\
(\mathrm{mm})\end{array}$ & $\begin{array}{c}\text { Total } \\
\text { Elements } \\
\text { (Millions) }\end{array}$ \\
\hline \multirow{3}{*}{ DrivAer } & Coarse & 20 & 100 \\
& Medium & 10 & 150 \\
& Fine & 5 & 300 \\
\hline \multirow{3}{*}{ Large Saloon } & Coarse & 20 & 150 \\
& Medium & 10 & 200 \\
& Fine & 5 & 350 \\
\hline \multirow{3}{*}{ SUV } & Coarse & 20 & 300 \\
& Medium & 10 & 350 \\
& Fine & 5 & 450
\end{tabular}

Page 5 of 19 

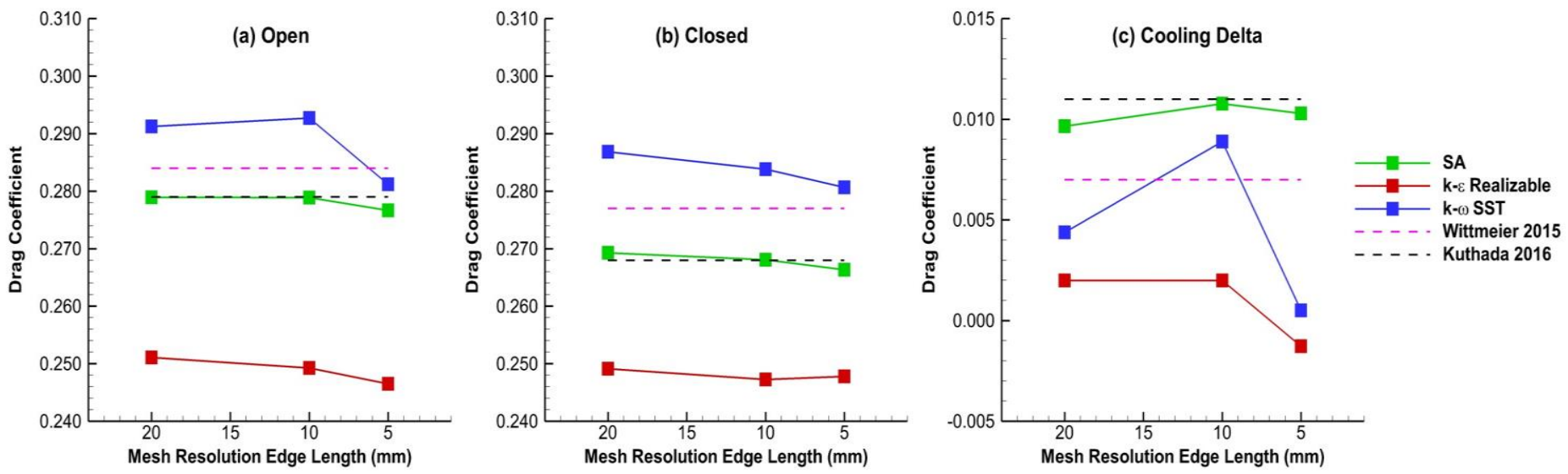

Figure 8. Grid convergence plots for open and closed cases for the three RANS models for the DrivAer case, alongside referenced experimental work, plotted as a straight dashed line.

\section{Results}

This section includes results from the two areas of study. Firstly, results will be presented for the RANS study of three turbulence models on three levels of grid refinement. This enables the investigation into cooling drag deltas and whether conclusions of previous work on the large saloon with the $\mathrm{k}-\varepsilon$ realizable [5], which proved successful in capturing this value, transfer to other car shapes and whether it is the best turbulence model to capture the whole flow field, i.e. whether the right answers were obtained for the right reasons.

This will be followed by supplementary comparative simulations on the DrivAer model using the two eddy resolving transient methods, namely the IDDES and LBM solvers, using industry best practices. These will act as validation for the DrivAer model, since there is currently no reference data available for the current set up: full scale 'open road' cooling notchback CAD with rotating wheels, moving ground, zero leak cooling package and tangential wheels (i.e. no contact patches).

\section{RANS}

The three car models will be presented separately with the DrivAer model followed by the full-production vehicles. For each model force coefficients will be analysed for the various turbulence models and grids in both open and closed configurations.

\section{DrivAer}

The presented drag and lift coefficients are calculated using a measured frontal area for the full-scale DrivAer of $2.17 \mathrm{~m}^{2}$. The results are compared to the initial experimental results of Wittmeier et al [12] and the secondary results of Kuthada et al [13]. These will act as a guideline but quantitative error will not be computed due to the differences in set-up which were outlined in the previous sections. The difference in cooling drag deltas between the two previous experimental works was attributed to an adjustment to the radiator simulators pressure drop.

\section{Drag}

Figure 8 shows the findings of the grid convergence study on the drag coefficient for the RANS turbulence models. Also plotted are straight lines indicating the reference experimental values from the previous DrivAer cooling work [13]. Plot (a) in Figure 8 shows the results for the open cooling case while plot (b) displays the closed grille case, (c) shows the difference, or cooling delta, between the open and closed cases.

From Figure 8a, for the open cooling case, it can be seen that the k- $\varepsilon$ realizable and SA turbulence models are relatively mesh independent for this vehicle, as $C_{d}$ only varies by a few counts. The SA model gives good agreement for absolute drag in comparison to the most recent work of Kuthada et al. The k- $\varepsilon$ realizable model is quite a bit lower than the other RANS models. It can be seen that the grid refinement has a positive effect on the k- $\omega$ SST model whose absolute drag values converge towards the others. This is the first indication that the previous work using the $\mathrm{k}-\varepsilon$ realizable could have been a result of a favorable predictive capability for that particular geometry as opposed to being a generally applicable validation of the methodology for automotive flows.

In the closed simulations, shown in Figure 8b, the models follow a similar pattern with the $\mathrm{k}-\varepsilon$ realizable predicting lower drag while the $\mathrm{SA}$ again gives a very similar value to the previous reference work. The k- $\omega$ SST model in this case tends towards the SA and reference data however on the finest mesh it is still 10 counts $\left(C_{d}=0.010\right)$ higher representing a percentage difference of $+3.7 \%$. This is similar to differences seen by Ashton et al [16] on the fastback model of $+2.3 \%$ and on the estate model of $+7.1 \%$.

The implication of the higher closed value for the absolute drag coefficient using the k- $\omega$ SST can be seen in Figure 8c, showing the cooling drag deltas. In Figure $8 \mathrm{c}$, it can be seen again that on all meshes the SA model does a good job of correctly predicting the value with respect to the most recently referenced work, with the same cooling package. While the k- $\omega$ SST model trends towards the SA and reference data on the medium mesh the finest mesh reports a much smaller cooling delta, the result of the high closed case absolute drag coefficient. The k- $\varepsilon$ realizable model however reports only a few counts of cooling drag on the coarser meshes and similarly a negative cooling drag value on the finest mesh.

From the grid convergence plots it can be seen that the cooling drag deltas for the DrivAer model in this particular set-up are sensitive to the RANS turbulence model used. Displayed in Figures 9 and 10 are the drag development plots from the finest meshes for the open and closed geometries, where the differences begin to occur. 


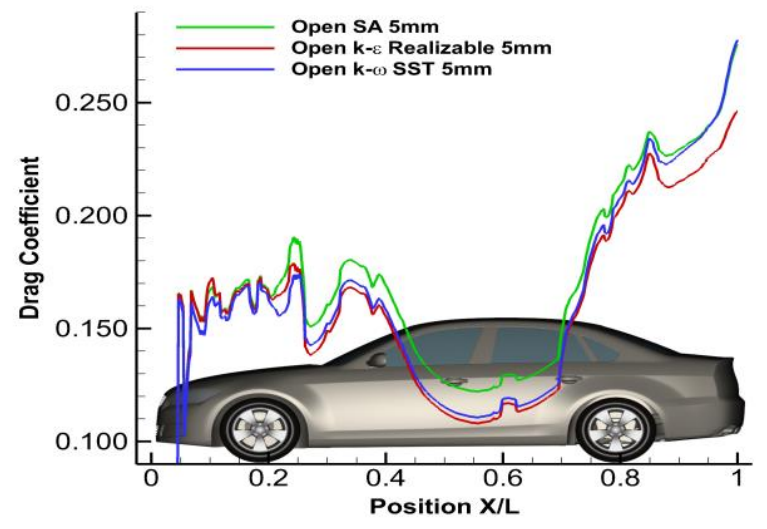

Figure 9. Drag development plot for the RANS models, indicating the area of divergence to the rear of the front wheels for the open cooling configuration.

All models are very similar in their predictions up to the front wheels $\mathrm{x}$-location $(\mathrm{X} / \mathrm{L}=0.2)$. Divergences between all the models in both open and closed configurations occur at $x$-locations near the rear of the front wheels. This results in a lowering of the drag development curve for the k- $\varepsilon$ realizable model in both open and closed configurations. In the open case, whilst both the SA and k- $\omega$ SST finish with similar absolute values the drag builds up at different $\mathrm{x}$ locations, which appears to be at x-locations coinciding with the front and rear wheels, suggesting while the values are the same it may not necessarily be accurate.

Figure 11 shows the breakdown of drag development for each wheel. It can be seen that the drag differences for the whole body are mirrored by the trends just over the wheels. This is important confirmation as drag development plots cannot differentiate between sources at the same x-location (e.g. cooling pack / wheels).

For the front wheel in the open configuration, it can be seen that the SA model's drag is more than 10 counts higher than the other two models at the rear of the wheel, it should be noted 50-60 counts absolute drag for the front wheels in the SA model is high. This is despite the fact that it has a very similar drag hitting the front of the tyre to that of the k- $\omega$ SST. In open and closed configurations a similar level of drag (within 5 counts) should be seen. This phenomenon is seen in both the SA and k- $\varepsilon$ realizable models however for the k- $\omega$ SST model the delta between the open and closed configuration for the front wheel is much larger (20 counts vs $<5$ counts). The questions to answer are whether the drag in the open or closed case is more realistic for the k- $\omega$ SST, and why is there an imbalance.

The large drag on the front wheels for the SA model shows why it is higher for the overall drag plot in Figure 9 between the front and rear wheels. However, it picks up less drag immediately upstream of the rear wheels unlike the other two models, suggesting the front wheel wake structures are different.

The front wheels in the closed configuration show a very similar drag development plot over the entirety of the front wheels using the SA and k- $\omega$ SST model. This could suggest for the DrivAer geometry in the closed case there are high pressure gradients that both RANS models struggle with. Whereas in the open configuration, in the presence of smaller pressure gradients, the k- $\omega$ SST model on the finest grid can more accurately predict the flow behavior.

Page 7 of 19

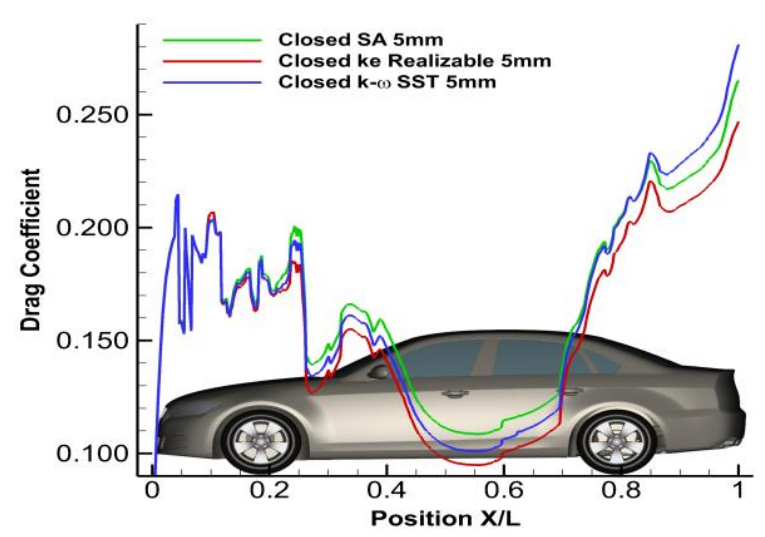

Figure 10. Drag development plot for the RANS models for the closed case.

For the rear wheels there are not such obvious differences. In both open and closed configurations the drag on the front of the tyre is very similar. In the closed case there are roughly 1-2 counts of variation between the drag predicted for the entire rear wheels. In the open case the drag of the rear wheel for the k- $\omega$ SST model is higher than the other two models, which could be a result of an incorrectly modelled front wheel wake interacting with the rear wheels.

From the drag development plots it can be seen that both the SA and $\mathrm{k}-\omega \mathrm{SST}$ models struggle with the high pressure gradients in the closed case, however, they don't easily explain the small cooling delta in the $\mathrm{k}-\varepsilon$ realizable model.
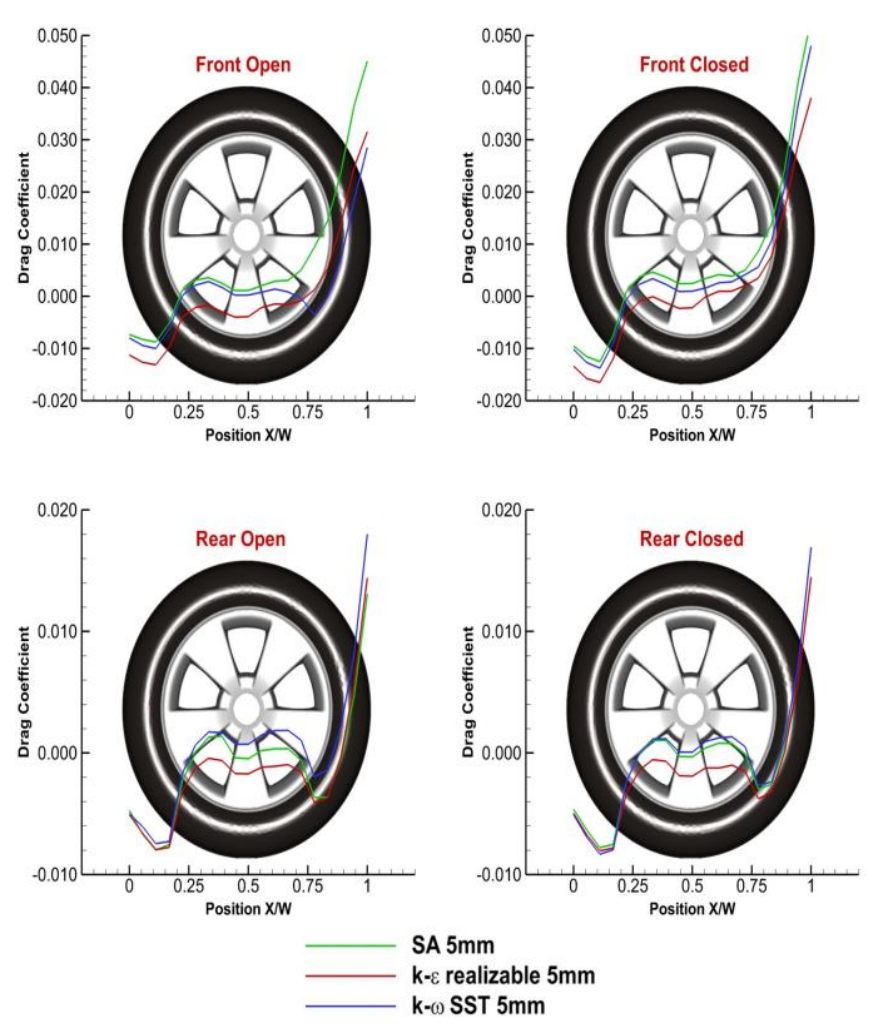

Figure 11. Drag development plots for front and rear wheels in both open and closed configurations for the three RANS models on the finest grid. 


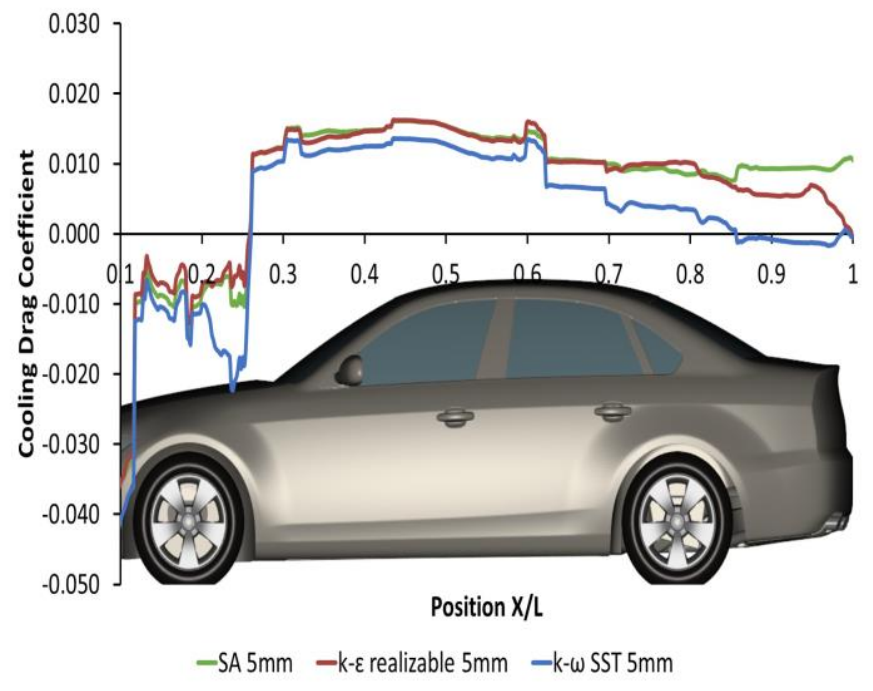

Figure 12. Cooling drag development plot showing the areas of divergence in the $\mathrm{k}-\omega \mathrm{SST}$ and $\mathrm{k}-\varepsilon$ realizable models in comparison to the SA turbulence model.

Figure 12, shows the cooling drag delta plots (open grille - closed grille) for the SA, k- $\varepsilon$ realizable and k- $\omega$ SST turbulence models. All turbulence models will have variations between them that cause slight differences in the absolute drag values they predict in automotive applications. However, in order to model cooling flow cases accurately, resolving the differences in the flow field and not just focusing on cooling drag as a function of cooling pack is of most importance.

Figure 12 shows that for the k- $\varepsilon$ realizable model despite a few peaks and troughs which could be attributed to the difference in the front $(\mathrm{X} / \mathrm{L}=0.25)$ and rear $(\mathrm{X} / \mathrm{L}=0.8)$ wheels, the difference between open and closed flow fields in the $\mathrm{k}-\varepsilon$ realizable and SA models is roughly the same up to $\mathrm{X} / \mathrm{L}=0.85$. The difference occurs near the base of the vehicle, between $\mathrm{X} / \mathrm{L}=0.95$ and $\mathrm{X} / \mathrm{L}=1$, where a sharp gradient can be seen without which, the $\mathrm{k}-\varepsilon$ realizable could have returned a much higher cooling delta.

The k- $\omega$ SST model and the SA models vary at $X / L=0.6$. At this $x-$ location there is little to separate the models and would suggest there is a difference in the structures of the front wheel wake interacting with the body and rear wheels. To the rear of the model, a plateau is seen between $(X / L=0.85$ and 1$)$, suggesting similar difference to the SA model.

Assessing the drag coefficient for the DrivAer test case has shown some interesting characteristics of cases with/without cooling flow and that of RANS turbulence models. With set-up difference caveats in mind, it would appear from simply looking at the absolute and cooling delta drag values of the SA turbulence model that it does an excellent job of modelling the test case in comparison to the reference experimental data. For the other two models no amount of grid refinement appeared to allow the models to correctly predict the flow fields in both configurations. The absolute drag coefficient in the open configuration, using the k- $\omega$ SST model, was shown to be within 1 count of the experiment, however in the closed configuration the drag over the front wheels was over predicted.

It has also been seen that when comparing the $\mathrm{k}-\varepsilon$ realizable and SA turbulence models the difference in cooling drag was attributed to the rear of the vehicle, potentially due to rear wheel wake interaction with the base wake or differences in modelling separation over the rear window. This highlights that despite similar drag values from the cooling package seen in Figure 9, the cooling deltas can be very different due to the importance of modelling the complex flow interactions around the model.

\section{Cooling Air Flow}

The cooling air coefficient, seen in Equation 2, is a measure based on the radiator area $\left(A_{R}\right)$, in this set-up of $0.35 \mathrm{~m}^{2}$, the freestream velocity $\left(v_{\infty}\right)$ and the cooling air volume flow rate $(\dot{V})$. It is a useful measure as it will validate the assumptions made in the drag coefficient section, stating the pressure drop remains unaffected despite large differences in the cooling drag delta.

$$
C_{V R}=\frac{\dot{V}}{v_{\infty} \times A_{R}}
$$

From Table 2 it can be seen there is very little difference between the models for the volume air coefficient travelling through the radiator on the finest mesh. They all have a good agreement in comparison to the CFD results of Wittmeier et al [12], while noting there is a difference in the porous media curve used in these simulations.

Also seen in Table 2 are the drag coefficient values of the porous media modelled radiators. Here it is seen that there is only 1 count variation between turbulence models despite up to 12 counts variation in the cooling drag delta.

Table 2: Table of Cooling Volume Air Coefficient for the RANS models presented for finest mesh.

\begin{tabular}{ccccc}
\hline & SA & $\begin{array}{c}\mathrm{k}-\varepsilon \\
\text { realizable }\end{array}$ & $\begin{array}{c}\mathrm{k}-\omega \\
\mathrm{SST}\end{array}$ & $\begin{array}{c}\text { CFD } \\
2015 \\
{[11]}\end{array}$ \\
\hline $\begin{array}{c}\text { Cooling Air } \\
\text { Coefficient }\end{array}$ & 0.087 & 0.088 & 0.086 & 0.096 \\
$\begin{array}{c}\text { Porous Media } \\
\text { Drag } \\
\text { Coefficient }\end{array}$ & 0.047 & 0.048 & 0.047 & N/A \\
\hline
\end{tabular}

Figure 13 details the cooling drag split (\%) through the grilles and out through the wheel arches and underbody to the nearest whole integer for the RANS model on the finest mesh in the open configuration. Obtained by integrating the flow velocities through the outlets.

The initial results of Wittmeier et al [12], showed a 60-40 split for the lower and upper intake grilles respectively. All three RANS models agreed, within $2 \%$, that the split was similar, the small difference could be explained due to the different set-up or differences between the Navier-Stokes methods used for RANS and the LBM method used in the previous work.

The previous work of Wittmeier et al [12] found that $3 / 4$ of the exiting flow went through the wheel arches. In this set up with much smaller circular apertures, the balance between wheel arch exit and underbody exit is $45-55 \%$ respectively. Similarly, in the previous work a right-left imbalance (14\%) was noted in the flow exiting from the wheel arches. Here, it has been found that they are within $1 \%$. 


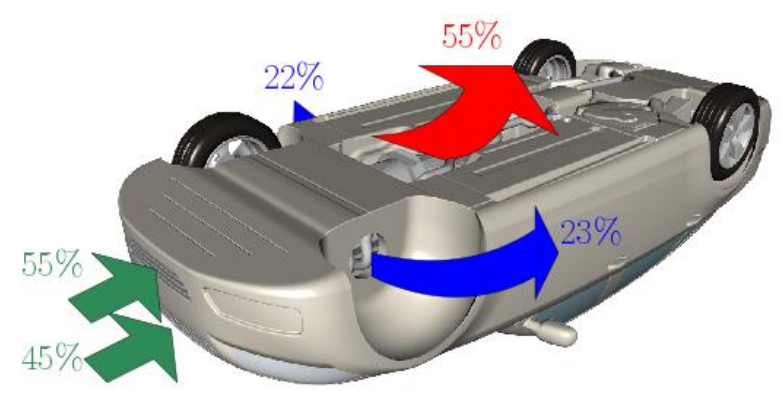

Figure 13. Cooling air split of the SA turbulence model on the finest grid.

\section{Lift}

The lift coefficient is derived by computing the moment coefficient about the front $\left(C_{L R}\right)$ and rear $\left(C_{L F}\right)$ axles, using the wheelbase $(2.8 \mathrm{~m})$ as the reference length. Figure 14, shows the direction and position of the lift forces on the front and rear axles for the DrivAer model

Presented in Table 3 are the results from the finest mesh alongside the reference experimental results of Kuthada et al [13]. Also presented are the drag coefficient results to allow the identification of trends between the force coefficients

It can be seen that in this set up the magnitude of the lift is over predicted compared to the previous experiment. Studying the convergence with mesh resolution, not shown, suggests all RANS models converge to the finest mesh values except for the $\mathrm{k}-\varepsilon$ Realizable model, mirroring the drag coefficient, which is mesh independent.

By studying the lift coefficient as well as the drag, patterns start to emerge. The lift coefficient of the k- $\omega$ SST model is very close in value to the SA model for all components of lift. It also shows that while the $\mathrm{k}-\varepsilon$ realizable model which previously has been used on full-production vehicles differs from the other two RANS turbulence models, it would appear that k- $\varepsilon$ realizable's robustness causes it to average out flow oscillations (unsteadiness) that occur in the SA and k- $\omega$ SST models.

Table 3: Table of drag and lift absolute values alongside experimental values from Kuthada et al [13].

\begin{tabular}{ccccc}
\hline \multirow{2}{*}{ Model } & Configuration & $C_{d}$ & $C_{L F}$ & $C_{L R}$ \\
& & & & \\
\hline \multirow{2}{*}{ SA } & Open & 0.277 & -0.076 & 0.107 \\
& Closed & 0.266 & -0.100 & 0.121 \\
\hline \multirow{2}{*}{ k- $\varepsilon$} & Open & 0.247 & -0.097 & 0.084 \\
realizable & Closed & 0.248 & -0.104 & 0.115 \\
\hline \multirow{2}{*}{ k- $\omega$ SST } & Open & 0.281 & -0.073 & 0.121 \\
& Closed & 0.280 & -0.113 & 0.123 \\
\hline \multirow{2}{*}{ Experimental } & Open & 0.278 & -0.014 & 0.068 \\
& Closed & 0.267 & -0.039 & 0.098 \\
\hline
\end{tabular}

Page 9 of 19

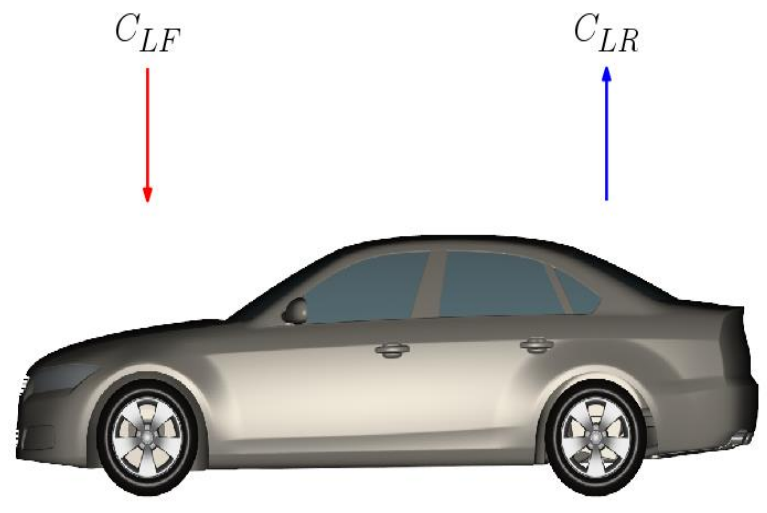

Figure 14. Lift at the front $C_{L R}$ and rear $C_{L F}$ axle.

\section{Large Saloon}

The presented large saloon is the same as seen in previous work by Simmonds et al in 2016 [5]. The previous work was modelled using the coarsest grid $(20 \mathrm{~mm})$ and the $\mathrm{k}-\varepsilon$ realizable model which obtained an accurate value of the cooling drag delta. This model does not have a wealth of experimental data but is included as the absolute drag values are known. The model therefore provides a further test of the grid convergence study of the DrivAer model and whether further refinement of the k- $\omega$ SST and SA model will improve the wake and drag coefficient. It will also act as a comparison to see if similar trends are seen between the turbulence models.

During this paper a slightly different approach was used in wrapping the large saloon and SUV surfaces in comparison to the previous work of Simmonds et al [5]. Initially a very fine detail wrap was applied and then the aero-surfaces were replaced with the manually meshed surfaces from the original CAD models. A side-by-side comparison of the two wrapping methods can be seen in Figure 16. This comparison was achieved by using the old surface in the current set-up by removing fan-rotation and heat transfer from the cooling pack, and running at the current work's freestream velocity.

Figure 16 shows the drag development plots for the k- $\varepsilon$ realizable model using the coarsest $(20 \mathrm{~mm})$ mesh settings. Plotted on the primary axis (shown on the left) is the delta between the old and new surface definitions. The actual drag development plots are plotted in red and blue but for neutrality and confidentiality the axis has been removed, as the purpose is to outline the regions of divergence between the old and new surfaces.

Both of the delta show fairly similar patterns between the open and closed surfaces. It shows that the effect of the improved definition has reduced the drag coefficient on the new surfaces by 11 counts in the open configuration and 17 counts in the closed case. It can be seen over the front of the model more drag is picked up on the new model. Initially it would appear it could be due to the improved definition in the engine-bay, based on the x-location, however the closed configuration sees a larger difference suggesting that it is the aero-surfaces where high-pressure gradients are experienced around the front end. This is supported since the closed case sees the largest overall difference from the old surfaces. It could be suggested that the coarser definition adds artifacts (bumps) near joins, such as between the a-pillar and windscreen, that increases the drag seen on the older coarser surface. The new surface also improves on the prediction of 

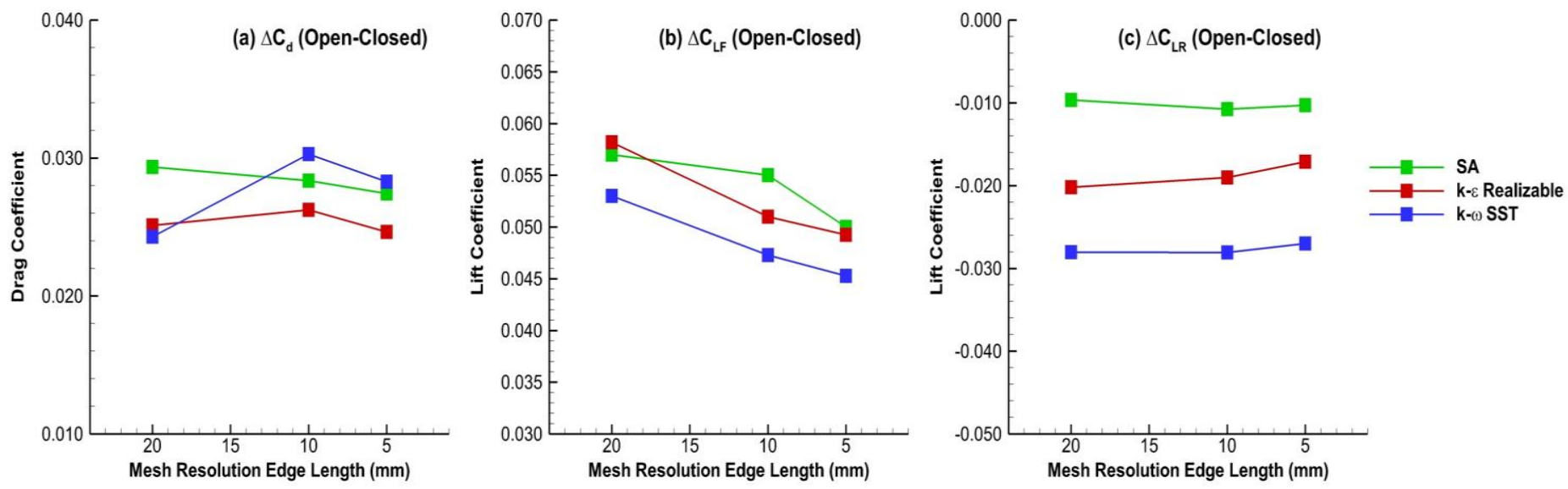

Figure 15. Cooling delta grid convergence plots for lift and drag coefficients for the three RANS models for the Large Saloon test case.

the cooling drag delta. The 6 count swing that both surfaces undergo means the new cooling delta is 25 counts, which perfectly matches the wind tunnel, rather than 19 seen on the old surface in the current set-up

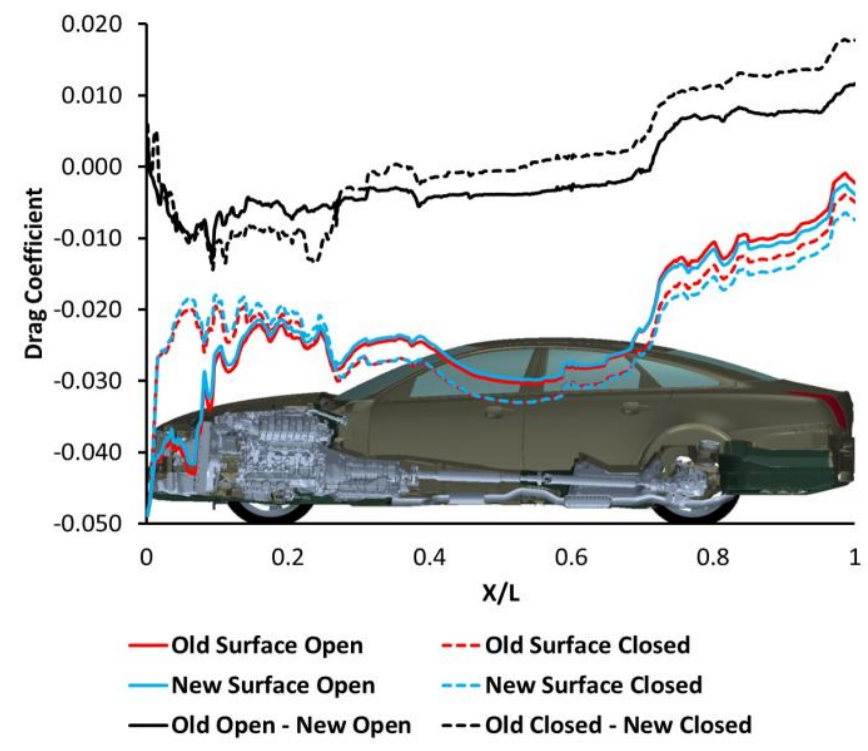

Figure 16. Drag development plot comparing the coarse vs updated wrapping procedures for the aero-surfaces on the large saloon using the $\mathrm{k}-\varepsilon$ realizable model with the coarsest $(20 \mathrm{~mm})$ mesh settings

It is worth noting however that the improved surface definition has doubled the number of surface elements before volume meshing. Despite the increase in surface elements it is highly recommended in all future cooling flow assessments for a very detailed wrap of the underhood components on a full production car in order to accurately model the flow fields.

The grid convergence plots for lift and drag coefficient deltas using the new surface (current work wrap settings) can be seen in Figure 15. During the DrivAer section it was seen that the models experienced large variations in cooling deltas between the turbulence models and mesh sizes. Some of the differences were larger than the cooling delta itself, i.e. the k- $\varepsilon$ realizable model recorded a value 12 drag counts different from SA model, while the expected cooling delta was only 11 counts.

From the plots in Figure 15 it can immediately be seen that there is much more agreement between turbulence models, the maximum difference in drag delta between models is 5 counts, and that mesh refinement has less of an effect. It is also worth noting that the k- $\omega$ SST model, which varied largely with mesh refinement in the open case on the DrivAer model only changed by 3 counts for the cooling delta between the coarsest and finest mesh for the full-production large saloon.

For the k- $\omega$ SST model on the DrivAer model it was seen for the medium and fine mesh settings that the drag was over-predicted on the front wheel in the closed configuration in comparison to that predicted in the open case. On the large saloon this doesn't happen as seen in Figure 17.

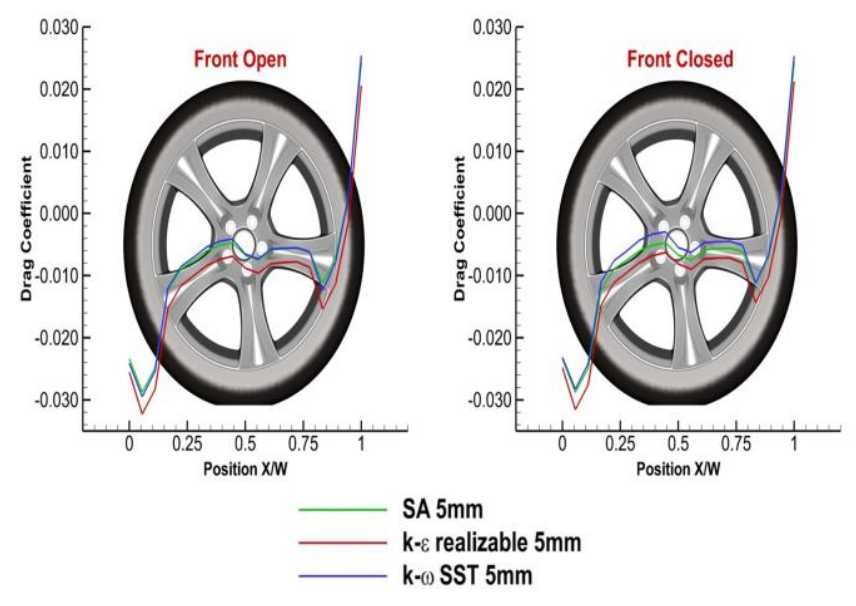

Figure 17. Drag development plots for front wheels in both open and closed configurations for the three RANS models on the finest grid for the large saloon.

Page 10 of 19 
Figure 17 shows the drag development plots for the front wheels on the large saloon in both open and closed configurations. It can be seen that all models agree within 1-2 counts for the overall drag which is much better than the 10-15 count variation seen on the DrivAer model. The large saloon also backs up the notion that the drag contribution from the front wheels should not change by a large amount (possibly just a few counts) between the open and closed configurations.

Further analysis of wheel drag between the large saloon and the DrivAer model would suggest that it is correct to say the SA model is over predicting drag. The front wheels' contribution to total drag on the large saloon is $\approx 10 \%$ whereas for the SA model in both configurations and the $\mathrm{k}-\omega \mathrm{SST}$ in the closed configuration drag contribution due to the front wheels was $>20 \%$.

The large saloon has shown that the improved surface wrapping technique is beneficial in not only correctly predicting the absolute drag but also cooling drag delta. It has also highlighted the differences seen between turbulence models on the DrivAer model were geometry specific and are not seen on this full-production large saloon. It would be suggested that the high pressure gradients on the front wheel of the DrivAer model, seen in Figure 18, greatly affects the front wheel drag and that RANS models, SA in particular struggles with the interaction between this and modelling wheel rotation. It is argued this phenomenon is not seen on the fullproduction large saloon due to the presence of the brake rotor, suspension and driving column which partially block the amount of flow capable of being drawn through the front wheels and the front wheel deflectors, seen in Figure 18. The large saloon also highlights that when using RANS models the difference between the drag accumulation on the front and rear wheels is quite small (0-5 counts) whereas on the DrivAer its very pronounced (10-30 counts) depending on the configuration

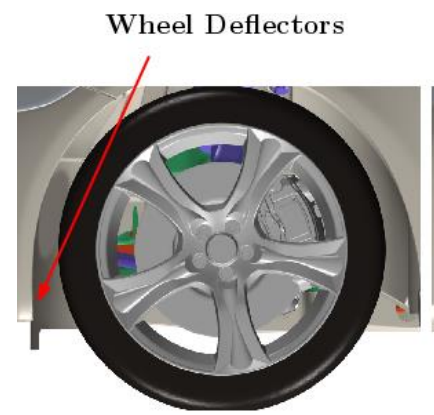

Large Saloon

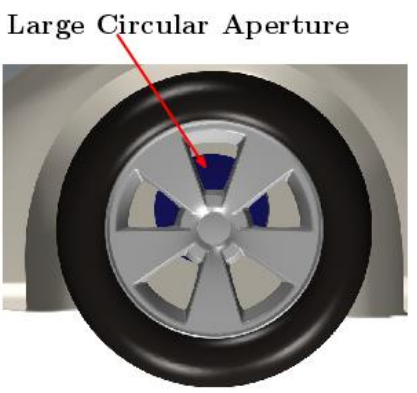

DrivAer
Figure 18 Difference in wheel arch geometry and leakages between the large saloon and DrivAer model, it can be seen the rotor partially blocks flow able to interact with the rotation of the spokes.

\section{SUV Results}

\section{Aerodynamic Coefficients}

Presented in this section are results obtained from the SUV. This model provides a very different modelling challenge to that of the notchback DrivAer and large saloon due to its large square-back bluff-body shape. Only presented are RANS simulations from the finest meshes due to the conclusions drawn in the large saloon section. It should be noted that while both the SA and k- $\omega$ SST have oscillations in the flow field, they are both mesh independent for this case similar to the large saloon and unlike the DrivAer cases.

Table 4 presents results from the simulations against the experimental values as an absolute difference. It can be immediately seen that of the three RANS models the k- $\omega$ SST gives the closest absolute drag values, calculated as within $2 \%$. It can also be seen that all models are fairly accurate when it comes to matching the frontal lift but for the rear lift they suffer large deviations, which is to be expected due to RANS inability to correctly predict large wake structures on similar automotive vehicles [16].

Table 4: Table of drag and lift deltas against absolute experimental values from experiment using FKFS wind tunnel

\begin{tabular}{ccccc}
\hline \multirow{2}{*}{ Model } & Configuration & $\Delta C_{d}$ & $\Delta C_{L F}$ & $\Delta C_{L R}$ \\
\hline \multirow{2}{*}{ SA } & Open & 0.015 & -0.015 & 0.098 \\
& Closed & 0.013 & 0.010 & 0.093 \\
\hline \multirow{2}{*}{ k- $\varepsilon$} & Open & 0.021 & -0.015 & 0.126 \\
realizable & Closed & 0.020 & 0.007 & 0.111 \\
\hline \multirow{2}{*}{ k- $\omega$ SST } & Open & -0.009 & -0.012 & 0.090 \\
& Closed & -0.007 & -0.017 & 0.087 \\
\hline
\end{tabular}

Table 5 presents deltas against the experimental values but in comparison to the cooling values, open grille results minus closed grille results, where $\Delta C_{d_{C}}=35$ counts. The takeaway point from the table is that all models can very accurately predict the value of the cooling drag delta, within 2 counts, which is in agreement with the other full-production model, the large saloon, but at odds with the notchback DrivAer. For the lift values, the k- $\omega$ SST model most accurately predicts the front and rear changes to lift when the grills are open and closed, however again it should be noted that lift values have proven much more difficult to predict both experimentally and computationally and the relative performance of the other two turbulence models is reasonable.

Figure 19 is a drag development plot of the SUV for the three RANS models seen in Tables 4 and 5 in both open and closed configurations; actual values are removed to avoid confidentiality issues. It can be seen that the front wheels in both configurations sustain little deviation as seen in the other full-production model. The deviation in this case occurs at the base of the vehicle providing further evidence of each models struggle with predicting the wake of the SUV

Table 5: Table of drag and lift deltas against cooling difference values from experiment using FKFS wind tunnel

\begin{tabular}{ccccc}
\hline Model & Refinement & $\Delta C_{d_{C}}$ & $\Delta C_{L F_{C}}$ & $\Delta C_{L R_{C}}$ \\
\hline $\begin{array}{c}\text { SA } \\
\text { k- } \varepsilon \\
\text { realizable } \\
\text { k- } \omega \text { SST }\end{array}$ & Fine & 0.000 & -0.015 & 0.012 \\
& Fine & -0.001 & -0.022 & 0.016 \\
& & 0.002 & -0.005 & -0.003
\end{tabular}




\section{Flow Field Unsteady Fluctuations}

The results from SUV test corroborate the ideas developed in the large saloon case. The wheel drag value trends seen on the large saloon are also seen on this full-production vehicle, opening up questions about the suitability of the DrivAer model in its current configuration to be used as a pseudo-full production vehicle since RANS models struggle with the flow features seen in the front wheel arches which are not present on the large saloon and SUV fullproduction vehicles tested here.

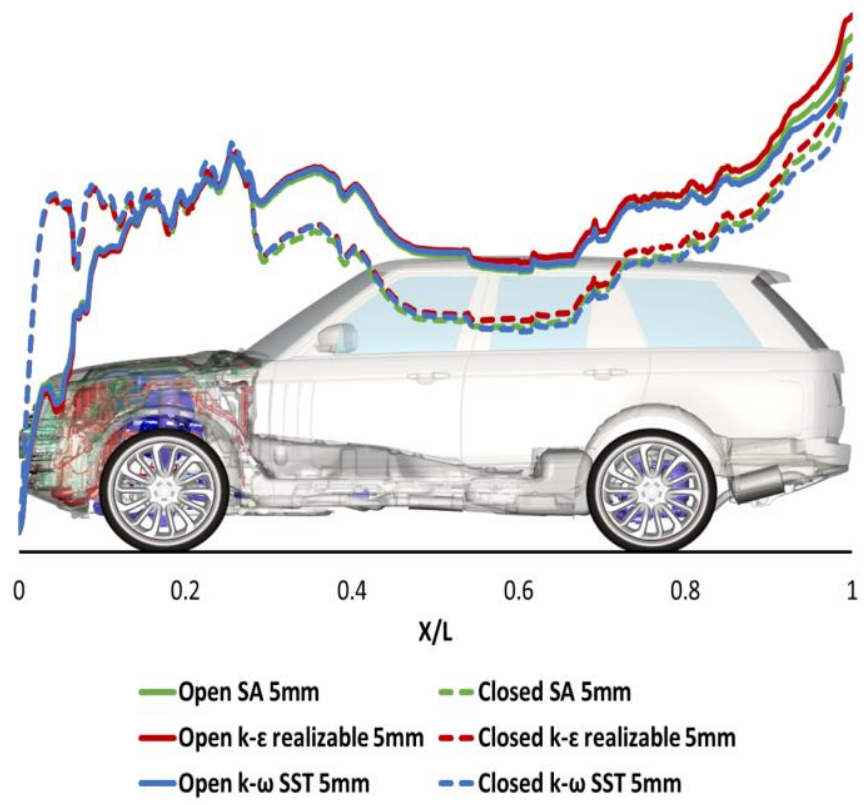

Figure 19. Drag development plot for the SUV showing the deviations between models occurs near the base of the vehicle.

\section{Eddy Resolving Transient Methods}

In this section the previously presented DrivAer model will be built upon in order to validate the RANS models force coefficient values and further explore why the DrivAer model experiences large differences between turbulence models for absolute values as well as the cooling drag delta, while in the large saloon they were not present and for the SUV only absolute values differed but cooling deltas were well captured.

The transient IDDES and LBM solvers will be used in this section with a comparison of the differences in their flow fields. For the IDDES solver both MRF's and the sliding mesh approach will be presented to bridge the gap between the steady-state RANS, which used MRF's and unsteady IDDES simulations, this could help explain why the RANS methods struggle in predicting the flow fields around the wheels.

Un-steady (U)RANS simulations were not wholly utilized in this work, except in the boundary layer of the IDDES simulations, since the intention was to run eddy-resolving methods to properly capture the larger scales of turbulence. It was also decided that since the presence of rotating wheels dictated that the time-step must be of a certain size that efficiency wise (U)RANS offered little benefit.

Page 12 of 19
As mentioned during the RANS analysis for the DrivAer model both the SA and k- $\omega$ SST turbulence models experienced fluctuations in force coefficients suggesting a pseudo-unsteadiness in the flow while the $\mathrm{k}-\varepsilon$ realizable model, which converged with residuals an order of magnitude smaller $10^{-4}$ did not.

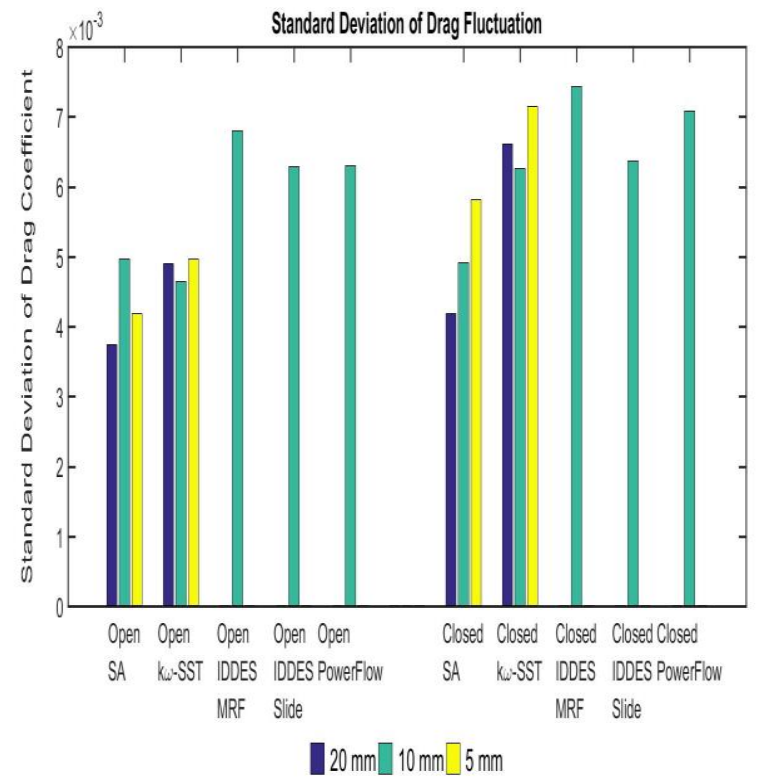

Figure 20. Standard deviation of drag fluctuations in the steady-state SA and $\mathrm{k}-\omega \mathrm{SST}$ models, showing similar levels when refined to those experienced in unsteady solvers.

Despite being a 1-equation turbulence model the SA model had a good agreement in both absolute values and the cooling drag delta when compared to the 2016 DrivAer experimental results, this section will assess whether this pseudo-unsteadiness is realistic.

Traditionally the SA and k- $\omega$ SST models have struggled in achieving convergence with methods recently being introduced with the aim of improving these problems [24]. However, in these simulations they both achieve satisfactory levels of convergence as outlined by the drag and lift residuals for all models seen in the Appendix. Shown are the final 10,000 iterations of the simulations, indicating that SA and $\mathrm{k}-\omega \mathrm{SST}$ are converged and that averaging over the final 5000 iterations is a suitable number to capture the pertinent flow characteristics presented in the paper.

In addition to the drag coefficient residuals, the standard deviations for the two fluctuating RANS models can be seen in Figure 20 in comparison to the unsteady simulation methods. It shows that as the grid density is increased for the RANS models, not only are the absolute drag values being refined but that the standard deviation experienced tends towards that seen in the transient simulations. It can also be seen that larger standard deviations are experienced in the closed cases, where higher pressure gradients are present, and that in the closed simulations the RANS models are closer. 
The work of Strangfeld et al [25] concluded experimentally that for the original fastback DrivAer geometry, without rotating wheels and a smooth underbody the maximum deviation from the mean force values were up to $5 \%$. The maximum standard deviation seen in Figure 20 is less than $3 \%$, while the maximum deviations for the finest mesh RANS models alongside the eddy-resolving methods can be seen in Table 6 .

Table 6: Table of maximum deviation from the mean drag force

\begin{tabular}{|c|c|c|c|}
\hline & Model & Configuration & $\begin{array}{c}\text { Maximum } \\
\text { Deviation \% }\end{array}$ \\
\hline \multirow{6}{*}{$\begin{array}{c}\text { Steady-State } \\
\text { RANS }\end{array}$} & \multirow{2}{*}{ SA } & Open & 2.42 \\
\hline & & Closed & 4.64 \\
\hline & \multirow{2}{*}{$\begin{array}{c}\mathrm{k}-\varepsilon \\
\text { realizable }\end{array}$} & Open & 0.15 \\
\hline & & Closed & 0.51 \\
\hline & \multirow{2}{*}{$\mathrm{k}-\omega \mathrm{SST}$} & Open & 4.80 \\
\hline & & Closed & 8.33 \\
\hline \multirow{6}{*}{ Transient } & \multirow{2}{*}{$\begin{array}{l}\text { IDDES } \\
\text { MRF }\end{array}$} & Open & 6.96 \\
\hline & & Closed & 6.69 \\
\hline & \multirow{2}{*}{$\begin{array}{l}\text { IDDES } \\
\text { Slide }\end{array}$} & Open & 6.90 \\
\hline & & Closed & 5.97 \\
\hline & \multirow{2}{*}{ LBM } & Open & 7.17 \\
\hline & & Closed & 7.92 \\
\hline
\end{tabular}

\section{Drag}

\section{Aerodynamic Coefficients}

Presented in this section are the results from the eddy resolving methods for lift and drag coefficient.

Figure 21 displays the absolute and cooling drag coefficient values for the LBM and two IDDES methods in open and closed configurations for the notchback DrivAer. It can be seen that both the LBM and IDDES method with sliding wheels achieve absolute values within a few counts of each and the experimental data. However, the cooling delta seen in LBM is half that seen in the IDDES simulations. The IDDES simulations which utilize the same wheel modelling technique as the steady state RANS simulations predicts a very good cooling delta, however the absolute values are 20 counts too high.

In a similar fashion to the RANS study, drag development plots for the three transient methods are presented in Figure 22 and 23. From both Figures it is seen that the LBM method predicts a higher stagnation drag on the front end of the vehicle in both the open and closed configurations, this difference is about 5 counts, suggesting it is a code-specific difference as opposed to a geometry specific one.
0.300

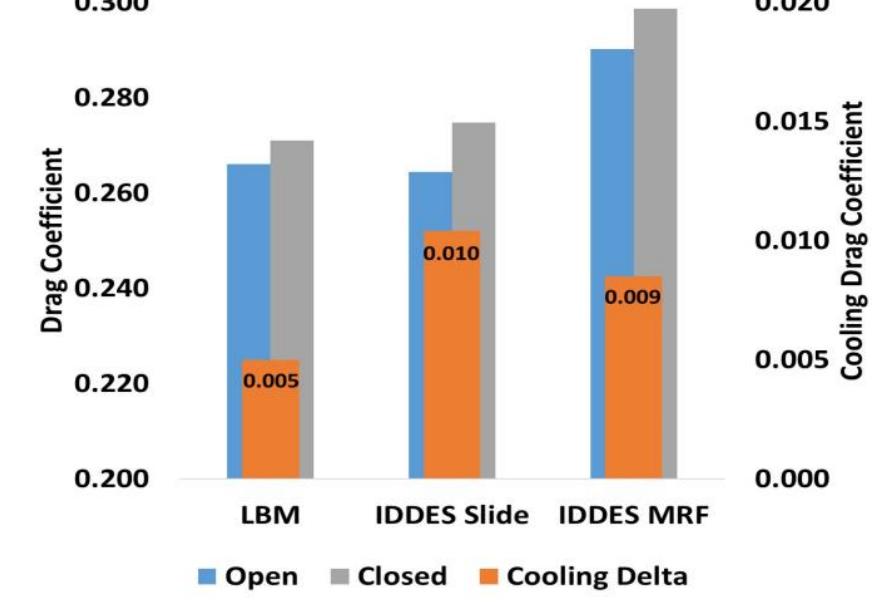

Figure 21 Absolute and cooling drag coefficient values for the LBM and two IDDES methods in open and closed configuration for the notchback DrivAer.

Despite the initial difference over the front end the IDDES slide and LBM methods are almost identical in both configurations from the rear axle to the base of the model. Both of the IDDES simulations pick up more drag at an $\mathrm{x}$-location immediately upstream of the rear wheels $(\mathrm{X} / \mathrm{L}=0.7)$, which may suggest the front wheel wake from the LBM solver is wider.

When comparing the two IDDES wheel modelling techniques it can be seen they are similar to the RANS simulations for areas of divergences. These occur to the rear of each set of wheels and in their wakes. For the MRF model the rear wheel wake created has a large effect on the base pressure which drives the absolute drag value much higher than seen in any other simulation technique. This interference however is seen in both the open and closed configurations, which indicates that while the wheel wake is not being correctly modelled it can predict the correct cooling delta, as seen in Figure 24.

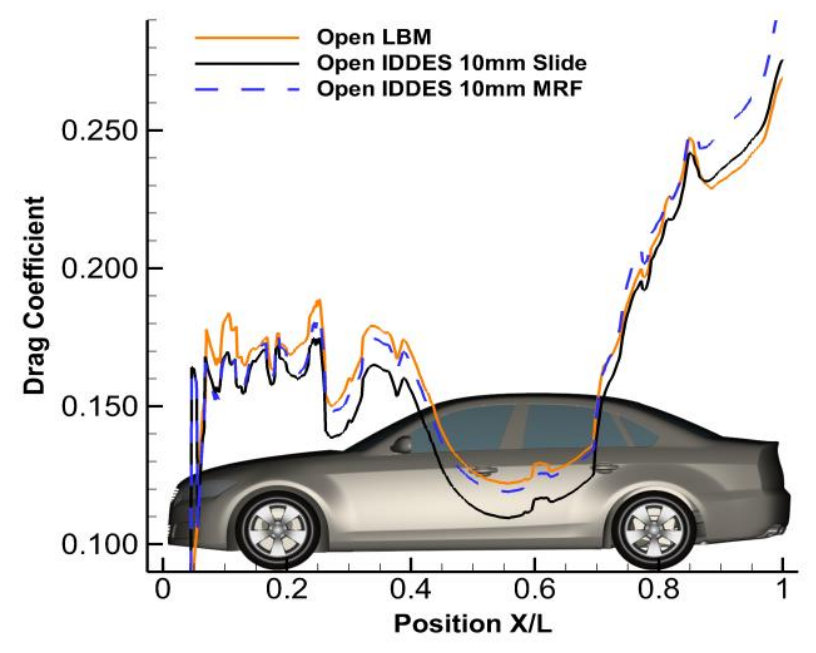

Figure 22. Drag development plot for the eddy-resolving models in the open DrivAer configuration, indicating the area of divergence at the front end between IDDES method and LBM and the rear wheels. 


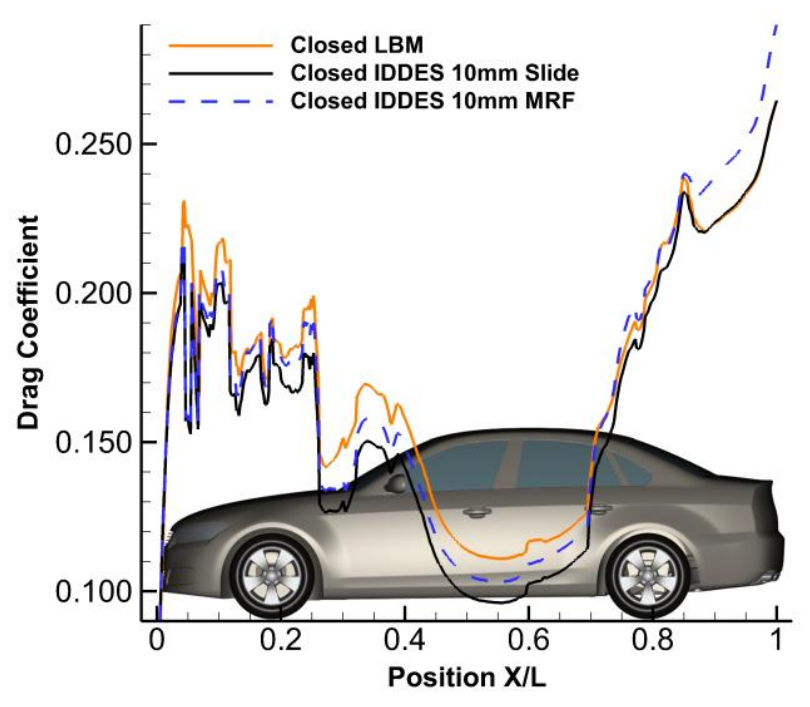

Figure 23. Drag development plot for the eddy-resolving models in the closed DrivAer configuration, indicating the area of divergence at the front end between IDDES method and LBM and the rear wheels.

Figure 24 plots the cooling drag curves from immediately upstream of the front wheels to the base of the model. The areas of divergence which have already been studied in the k- $\varepsilon$ realizable and k- $\omega$ SST RANS models can be seen. However, the plot outlines that in terms of cooling drag both IDDES methods and the SA RANS model all predict similar differences in the flow-field between open and closed configurations.

The eddy-resolving IDDES methods highlight the importance of predicting the whole flow field to see the differences in open and closed configurations. While both IDDES methods and the SA model can all correctly predict the so-called 'cooling drag delta'. Only the IDDES slide method and the LBM method get close in terms of predicting the lift cooling deltas as seen in Table 7.

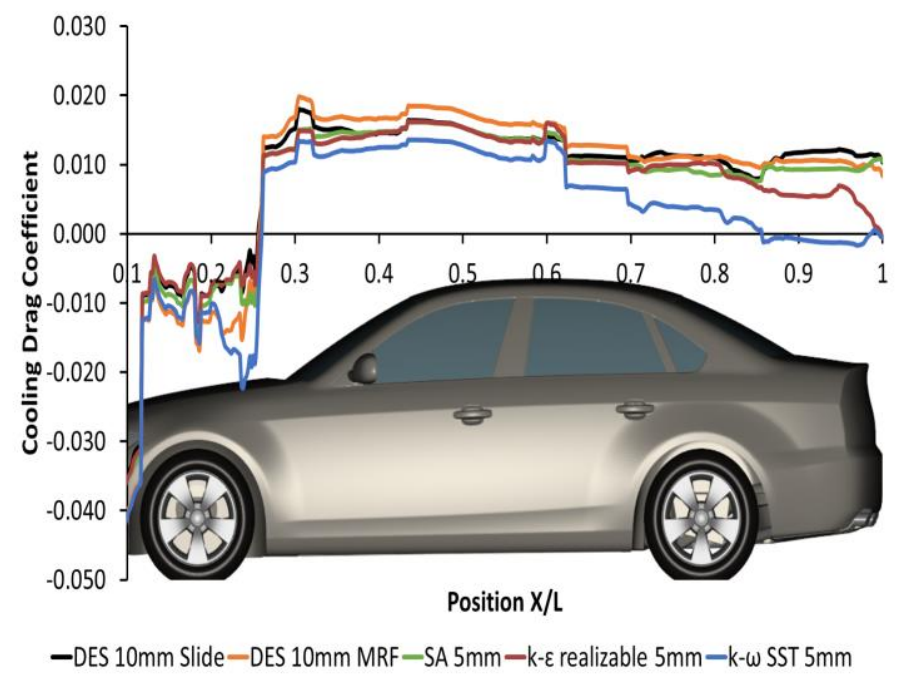

Figure 24. Drag development plot for the cooling deltas comparing the IDDES methods to the RANS simulations.

Page 14 of 19
Table 7: Table of drag and lift absolute values for Eddy Resolving methods alongside experimental values from Kuthada et al [13].

\begin{tabular}{ccccc}
\hline \multirow{2}{*}{ Model } & Configuration & $C_{d}$ & $C_{L F}$ & $C_{L R}$ \\
\hline \multirow{2}{*}{ IDDES MRF } & Open & 0.299 & -0.075 & 0.097 \\
& Closed & 0.290 & -0.123 & 0.121 \\
& Delta & 0.009 & 0.048 & -0.024 \\
\hline \multirow{3}{*}{ IDDES Slide } & Open & 0.274 & -0.089 & 0.086 \\
& Closed & 0.264 & -0.116 & 0.116 \\
& Delta & 0.010 & 0.027 & -0.030 \\
\hline \multirow{3}{*}{ LBM } & Open & 0.271 & -0.091 & 0.053 \\
& Closed & 0.266 & -0.115 & 0.084 \\
& Delta & 0.005 & 0.024 & -0.031 \\
\hline \multirow{2}{*}{ Experimental } & Open & 0.278 & -0.014 & 0.068 \\
& Closed & 0.267 & -0.039 & 0.098 \\
& Delta & 0.011 & 0.025 & -0.030 \\
\hline
\end{tabular}

It can be seen in Table 7 that the IDDES MRF method performs more similarly to that of the k- $\omega$ SST RANS models in terms of lift delta prediction. This adds further proof that the fluctuations found in the $\mathrm{k}-\omega$ SST RANS models are indicative of the unsteadiness found in the flow field due to the rotating wheels and highly unsteady wake. They differ in predicting drag at an X/L location of 0.25 , seen in Figure 24, where the cooling drag decreases sharply in the k- $\omega$ SST adding further evidence to its inability to predict the closed case correctly.
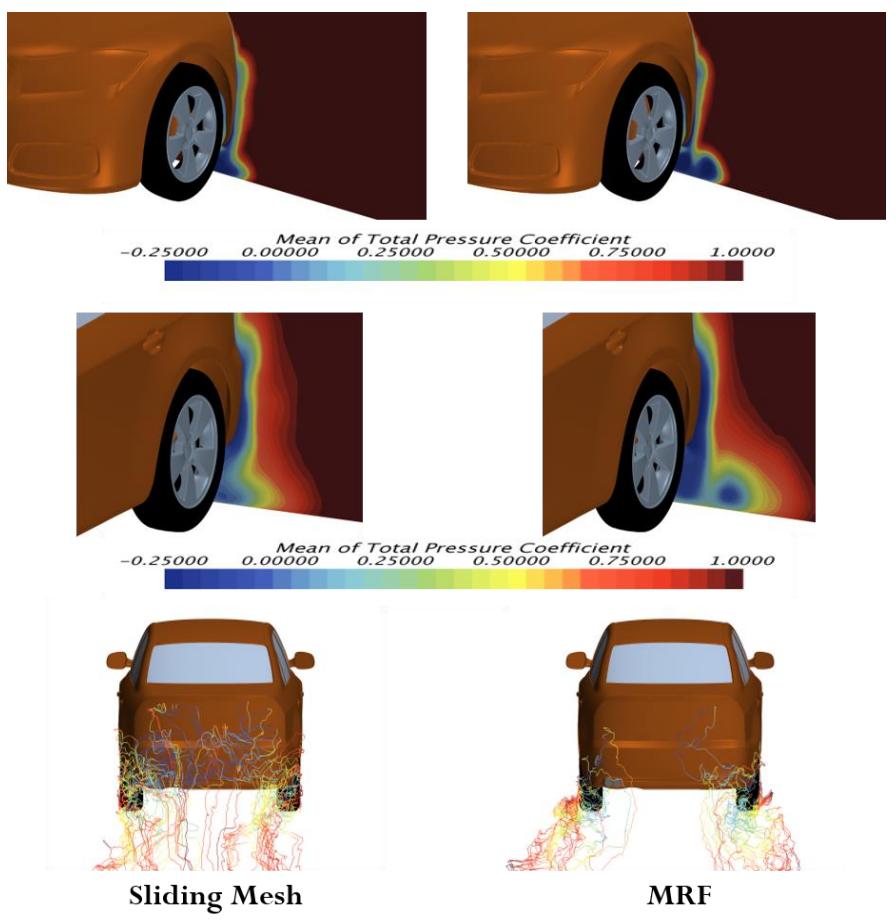

Figure 25. Side by side comparison of the two IDDES wheel modelling techniques and their effect on the wheel wake structures. 
Wheel drag prediction has proven to be one of the most important aspects of modelling the simple DrivAer geometry, in comparison to the full-production cars.

Figure 25 illustrates this by showing the wake structures of both the front and rear wheels using the IDDES solver with a sliding mesh and an MRF, in the closed configuration. The top image shows the front wheel wake, the middle image the rear wheel wake and the bottom image shows the rear wheel wake's interaction with the main wake of the model.

It can be seen that the wakes are pushed wider using the MRF technique particularly near the ground. From the rear wheels, the resultant wake changes the structure of the main body wake.

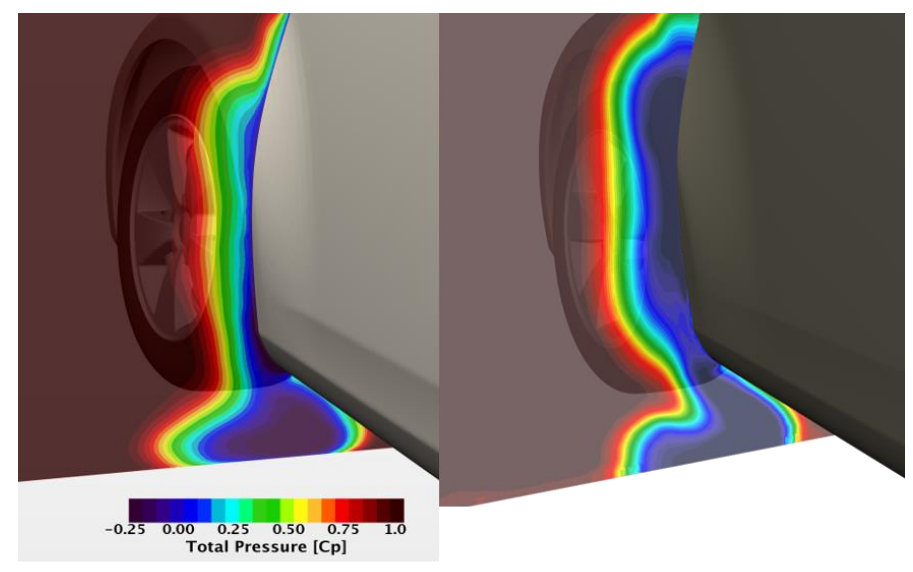

IDDES with Sliding Mesh

LBM

Figure 26. Side by side comparison of the IDDES and LBM methods for the front wheel wake.

Similarly, the differences between the sliding mesh models of IDDES and LBM can be compared. Seen in Figures 26 and 27 are the same front and rear wheel wake structures. Here the lower lobe structure from the front wheels are approximately the same size, however the upper lobe from the front wheels in the LBM case is much wider, which in turn could affect the flow onto the rear wheels.

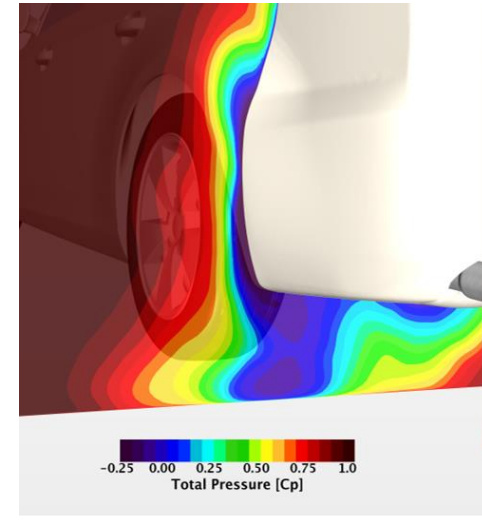

IDDES with Sliding Mesh

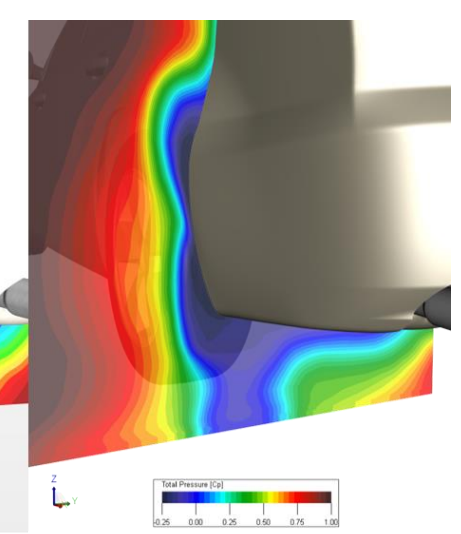

LBM
Figure 27. Side by side comparison of the IDDES and LBM methods for the rear wheel wake.

\section{Conclusions}

The aims of this paper were to discuss the notion of defining cooling drag as a number, to build upon already published literature of the DrivAer model and to develop connections to full production models in terms of modelling best practices.

Initially presented was a comprehensive review of three differently shaped vehicles, the Notchback DrivAer model and two fullproduction vehicles, a large saloon and a square-back SUV. This was undertaken using three RANS models on three different meshes in order to ascertain whether previous work done on the large saloon studying cooling flows could be replicated on other vehicle shapes.

For both the full-production vehicles, all three turbulence models were capable of predicting the cooling drag delta within 5 counts. On the large saloon between RANS turbulence models the absolute drag and lift values were also very consistent. The DrivAer model however was much more sensitive to both changes in turbulence models and mesh sizes, in agreement with previous work such as that of Ashton et al [17].

For the DrivAer model it appeared from simply looking at the absolute and cooling delta drag values of the SA turbulence model that it did an excellent job of modelling the test case in comparison to the reference experimental data. For the other two RANS models no amount of grid refinement appeared to allow the models to correctly predict the flow field. It also showed that using the k- $\omega$ SST model the drag count was predicted within 1 count of the reference experimental value in the open configuration, however there was a large imbalance between the open and closed front wheel drag predictions. It was suggested this was caused in the closed configuration. It was also seen that when comparing the $\mathrm{k}-\varepsilon$ realizable and SA turbulence models the difference in cooling drag was attributed to the rear of the vehicle, potentially due to rear wheel wake interaction with the base wake or differences in modelling separation over the rear window. This highlighted that despite similar drag values from the cooling package, the cooling deltas can be very different, suggesting that cooling drag cannot be thought of as openclosed drag with the addition of drag due to the cooling package.

Further work on the DrivAer model expanded on the RANS simulations utilizing the eddy-resolving methods, IDDES and LBM, as validation cases. The oscillations seen in the SA and k- $\omega$ SST RANS turbulence models were shown to be of similar levels to those in the transient methods indicating a pseudo-unsteadiness present in the steady-state solvers. Drag and lift coefficient absolute values were compared showing that only the IDDES method with sliding wheels and LBM method could obtain the closest results for the majority of the tested criteria. Further work should be undertaken studying the differences between the IDDES and LBM methods to ascertain whether the difference in the cooling delta which is present for the DrivAer is also seen on the full-production set-ups, and what causes this difference in the flow field, could help develop a new method for categorizing cooling flows.

The observations seen in the DrivAer model while furthering the discussion about modelling cooling flows and the need for a new measure to describe them, presents a new question which will require future research. This relates to connection between the DrivAer model and full-production cars, and it's use as a simplified fullproduction test-case. 


\section{References}

1. R. Barnard, N. Ledakis, Physical modeling and optimization of radiator cooling flow systems, in: Proc. 2nd MIRA Conference on Vehicle Aerodynamics, 1998.

2. R. H. Barnard, Theoretical and experimental investigation of the aerodynamic drag due to automotive cooling systems,

Proceedings of the Institution of Mechanical Engineers, Part D: Journal of Automobile Engineering 214 (8) (2000) 919-927. doi:10.1177/095440700021400810. URL http://pid.sagepub.com/lookup/doi/10.1177/0954407000214008 $\underline{10}$

3. A. Alajbegovic, B. Xu, A. Konstantinov, J. Amodeo, W. Jansen, Simulation of Cooling Airflow under Different Driving Conditions, SAE Technical Paper 2007-01-0766, doi:10.4271/ 2007-01-0766. URL http://papers.sae.org/2007-01-0766/

4. W. Jansen, J. Amodeo, E. Tate, Z. Yang, Drive cycle simulation of a tiered cooling pack using non-uniform boundary conditions, SAE Technical Paper 2014-01-0654, doi:10.4271/ 2014-010654.Copyright. URL http://papers.sae.org/2014-01-0654/

5. N. Simmonds, P. Tsoutsanis, A. Gaylard, W. Jansen, J. L. Rover, Full Vehicle Aero-Thermal Cooling Drag Sensitivity Analysis for Various Radiator Pressure Drops, SAE Technical Paper 2016-01-1578, doi:10.4271/2016-01-1578. URL http://papers.sae.org/2016-01-1578/

6. A. Gaylard, The Appropriate Use of CFD in the Automotive Design Process, SAE Technical Paper 2009-01-1162, doi: 10.4271/2009-01-1162. URL http://papers.sae.org/2009-01$\underline{1162 /}$

7. A. Alajbegovic, R. Sengupta, W. Jansen, Cooling Airflow Simulation for Passenger Cars using Detailed Underhood Geometry, SAE Technical Paper 2006-01-3478doi:10.4271/ 2006-01-3478. URL http://papers.sae.org/2006-01-3478/

8. W. Jansen, Engine Air Intake Thermal Modelling in Full Vehicle Underhood Environment, SAE Technical Paper 201301-0861, doi:10.4271/2013-01-0861. URL http://papers.sae.org/2013-01-0861/

9. C. Zhang, M. Uddin, X. Song, C. Fu, L. Foster, Simultaneous Improvement of Vehicle UnderHood Airflow and Cooling Drag Using 3D CFD Simulation, SAE Technical Paper 2016-010200, doi:10.4271/2016-01-0200. URL http://papers.sae.org/2016-01$\underline{0200 /}$

10. H. Soja, J. Wiedemann, The Interference between Exterior and Interior Flow on Road Vehicles, Ingenieures de l'Automobile 214 (1987) 101-105.

11. D. Baeder, T. Indinger, N. A. Adams, P. Unterlechner, G. Wickern, Interference Effects of Cooling Air-Flows with External Aerodynamics, International Journal of Automotive Engineering (2011) 115-121.

12. F. Wittmeier, T. Kuthada, Open Grille DrivAer Model - First Results, SAE International Journal of Passenger Cars Mechanical Systems 8 (1) (2015) 2015-01-1553, doi:10.4271/ 2015-01-1553. URL http://papers.sae.org/2015-01-1553/

13. T. Kuthada, F. Wittmeier, B. Bock, C. Schoenleber, A. Link, The Effects of Cooling Air on the Flow Field around a Vehicle, SAE Technical Paper 2016-01-1603, doi:10.4271/2016-011603. URL http://papers.sae.org/2016-01-1603/

14. T. Kuthada, J. Wiedemann, Investigations in a Cooling Air Flow System under the Influence of Road Simulation, SAE Technical Paper 2008-01-0796, doi:10.4271/2008-01-0796. URL http://papers.sae.org/2008-01-0796/

15. C. Collin, S. Mack, T. Indinger, J. Mueller, A Numerical and Experimental Evaluation of Open Jet Wind Tunnel Interferences using the DrivAer Reference Model, SAE International Journal of Passenger Cars - Mechanical Systems 9 (2) (2016) 2016-011597. doi:10.4271/2016-01-1597. URL

http://papers.sae.org/2016-01-1597/

16. E. Guilmineau, Numerical Simulations of Flow around a Realistic Generic Car Model, SAE International Journal of Passenger Cars - Mechanical Systems 7 (2) (2014) 646-653. doi: 10.4271/2014-01-0607. URL http://papers.sae.org/2014-01$\underline{0607 /}$

17. N. Ashton, A. Revell, Comparison of RANS and DES Methods for the DrivAer Automotive Body, SAE Technical Paper 201501-1538, doi:10.4271/2015-01-1538. URL http://papers.sae.org/2015-01-1538/

18. A. I. Heft, T. Indinger, N. Adams, Introduction of a New Realistic Generic Car Model for Aerodynamic Investigations, SAE Technical Paper 2012-01-0168, doi:10.4271/2012-010168. URL http://papers.sae.org/2012-01-0168/

19. P. R. Spalart, S. R. Allmaras, A one-equation turbulence model for aerodynamic flows, La Recherche Aerospatiale 1 (1) (1994) 5-21. doi:10.2514/6.1992-439.

20. T. H. Shih, W. W. Liou, A. Shabbir, Z. Yang, J. Zhu, A New Kepsilon Eddy Viscosity Model for High Reynolds Number Turbulent Flows: Model Development and Validation, Computer \& Fluids 24 (August) (1995) 227-238. doi:10.1016/0045-7930(94)00032-T.

21. F. R. Menter, Two-equation eddy-viscosity turbulence models for engineering applications, AIAA Journal 32 (8) (1994) 1598 1605. doi: $10.2514 / 3.12149$. URL http://dx.doi.org/10.2514/3.12149

22. D. C. Forbes, G. J. Page, M. A. Passmore, A. P. Gaylard, A Fully Coupled, 6 Degree-of-Freedom, Aerodynamic and Vehicle Handling Crosswind Simulation using the DrivAer Model, SAE International Journal of Passenger Cars - Mechanical Systems 9 (2) (2016) 2016-01-1601. doi:10.4271/2016-01-1601. URL http://papers.sae.org/2016-011601/

23. C. Landström, S. Sebben, L. Löfdahl, Effects of wheel orientation on predicted flow field and forces when modelling rotating wheels using CFD, in: 8th MIRA International Vehicle Aerodynamics Conference, 2010.

24. S.Evas, Lardeau.S, Validation of a turbulence methodology using the SST k- $\omega$ model for adjoint calculation, $54^{\text {th }}$ AIAA Aerospace Science Meeting, San Diego ,California, USA, 2016 doi:10.2514/6.2016-0585

25. Strangfeld, C., Wieser, D., Schmidt, H., Woszidlo, R. et al., "Experimental Study of Baseline Flow Characteristics for the Realistic Car Model DrivAer," SAE Technical Paper 2013-011251, 2013, doi:10.4271/2013-01-1251. URL http://papers.sae.org/2013-01-1251/

\section{Contact Information}

Nick Simmonds

Cranfield University

College Rd, Cranfield, Bedford MK43 0AL, UK

n.simmonds@cranfield.ac.uk

Page 16 of 19 


\section{Acknowledgments}

The authors would like to thank Jaguar Land Rover for granting permission to publish this work. They would also like to express thanks to Santhosh Jayaraju (JLR), for his assistance in running some of the models on the JLR HPC cluster

\section{Definitions, Acronyms, Abbreviations}

SUV: Sport Utility Vehicle

HPC: High Performance Computer

CFD: Computational Fluid Dynamics

RANS: Reynolds Averaged Navier Stokes

SA: Spalart-Allmaras Turbulence Model

SST: Shear Stress Transport

LES: Large Eddy Simulation

VLES: Very Large Eddy Simulation

DES: Detached-Eddy Simulation

IDDES: Improved Delayed Detached-Eddy-Simulation

LBM: Lattice Boltzmann Method

RNG: Re-Normalisation Group

CFL: Courant-Friedrichs-Lewy Number

MRF: Moving Reference Frame

$\boldsymbol{C}_{\boldsymbol{L}}: \quad$ Lift Coefficient Front

$\boldsymbol{C}_{\boldsymbol{L}}: \quad$ Lift Coefficient Rear

$\boldsymbol{C}_{\boldsymbol{d}}: \quad$ Drag Coefficient

$\boldsymbol{C}_{\boldsymbol{p}}$ : $\quad$ Pressure Coefficient 


\section{Appendix}

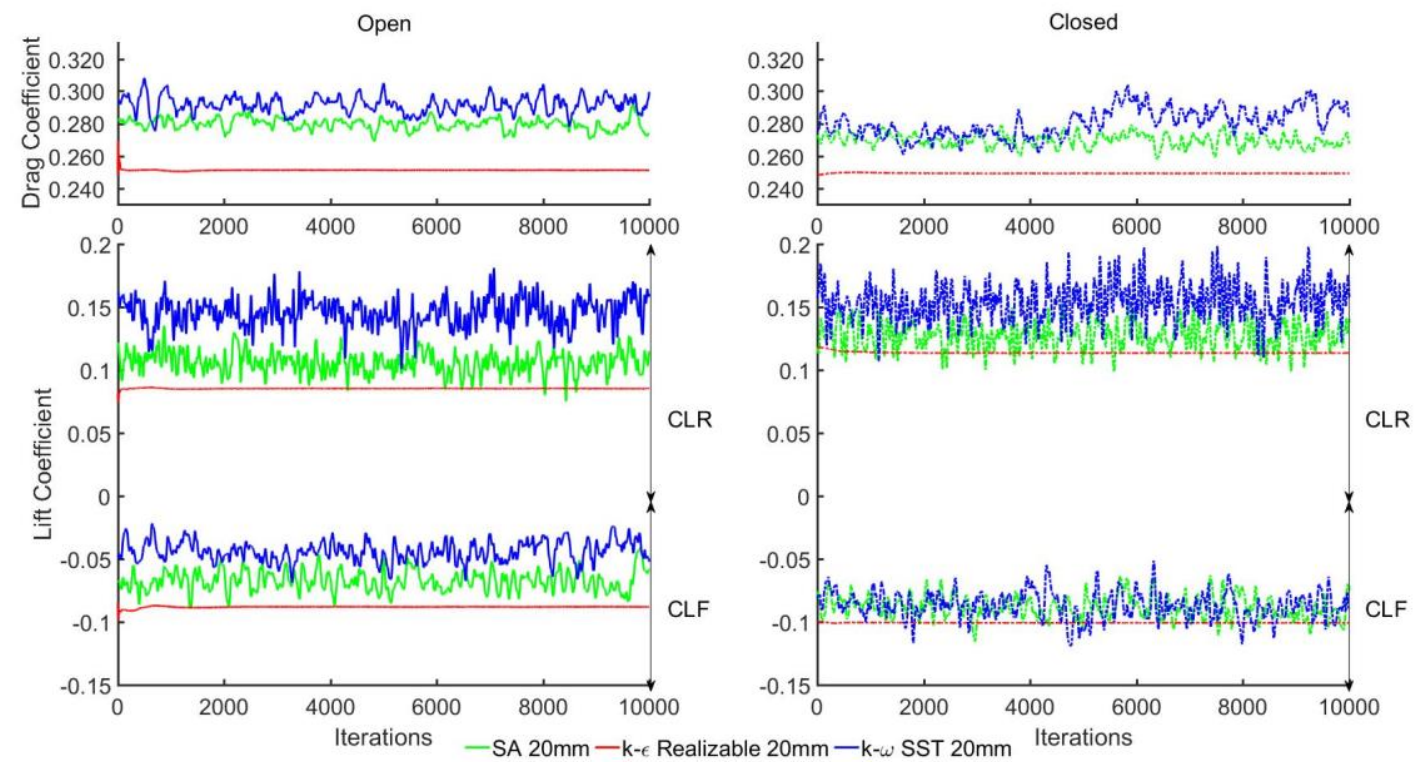

Figure 28. Lift and Drag residuals for the RANS models in open and closed configurations on the coarsest mesh

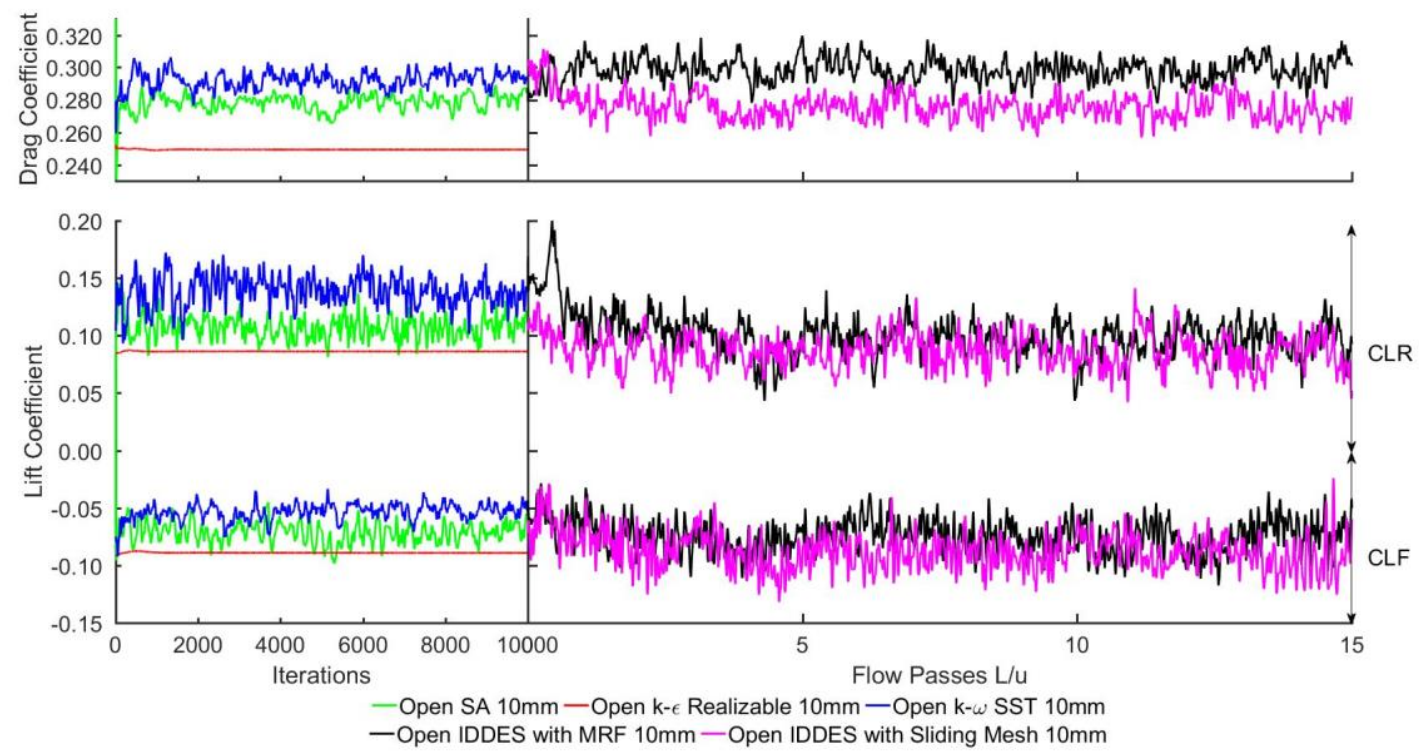

Figure 29. Lift and Drag residuals for the RANS and IDDES models in Open configurations on the medium mesh 


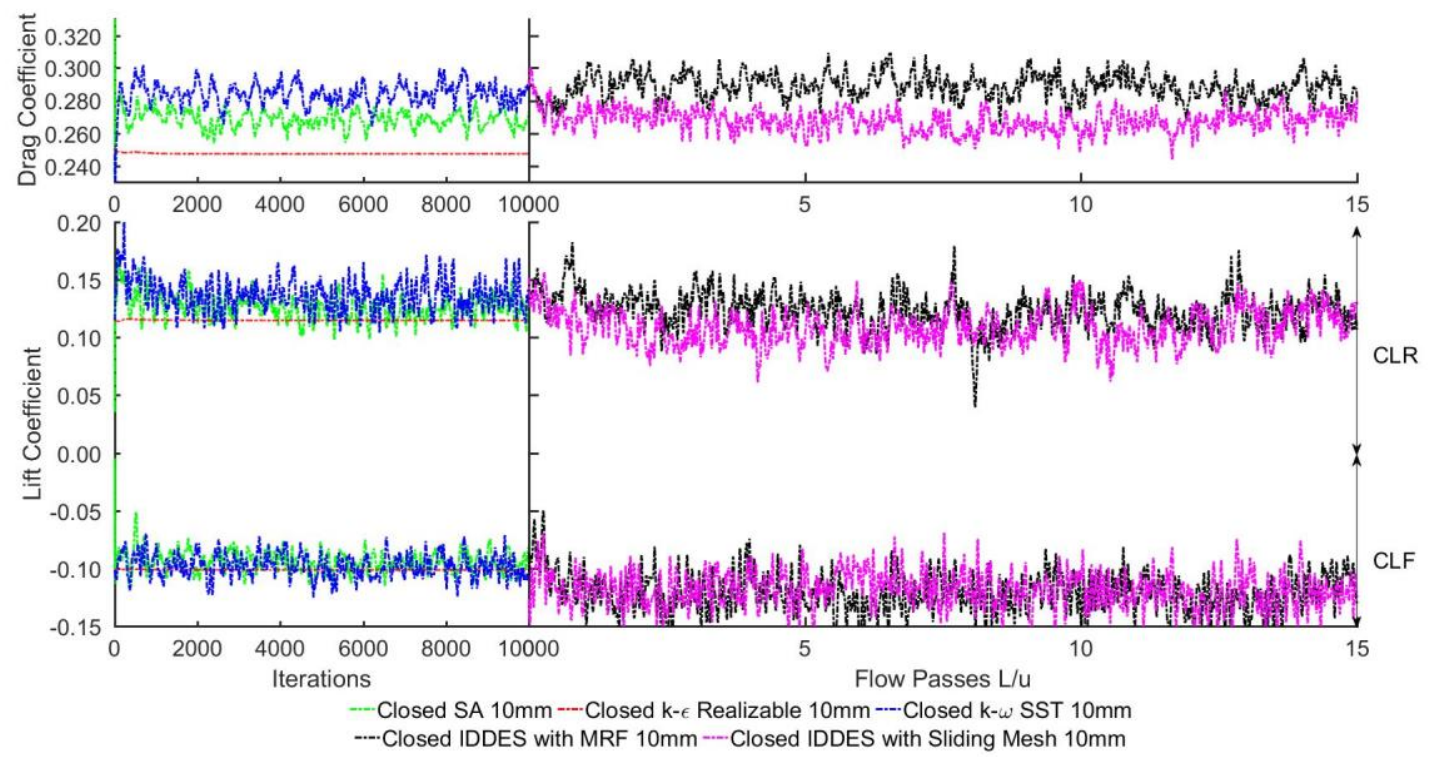

Figure 30. Lift and Drag residuals for the RANS and IDDES models in Closed configurations on the medium mesh

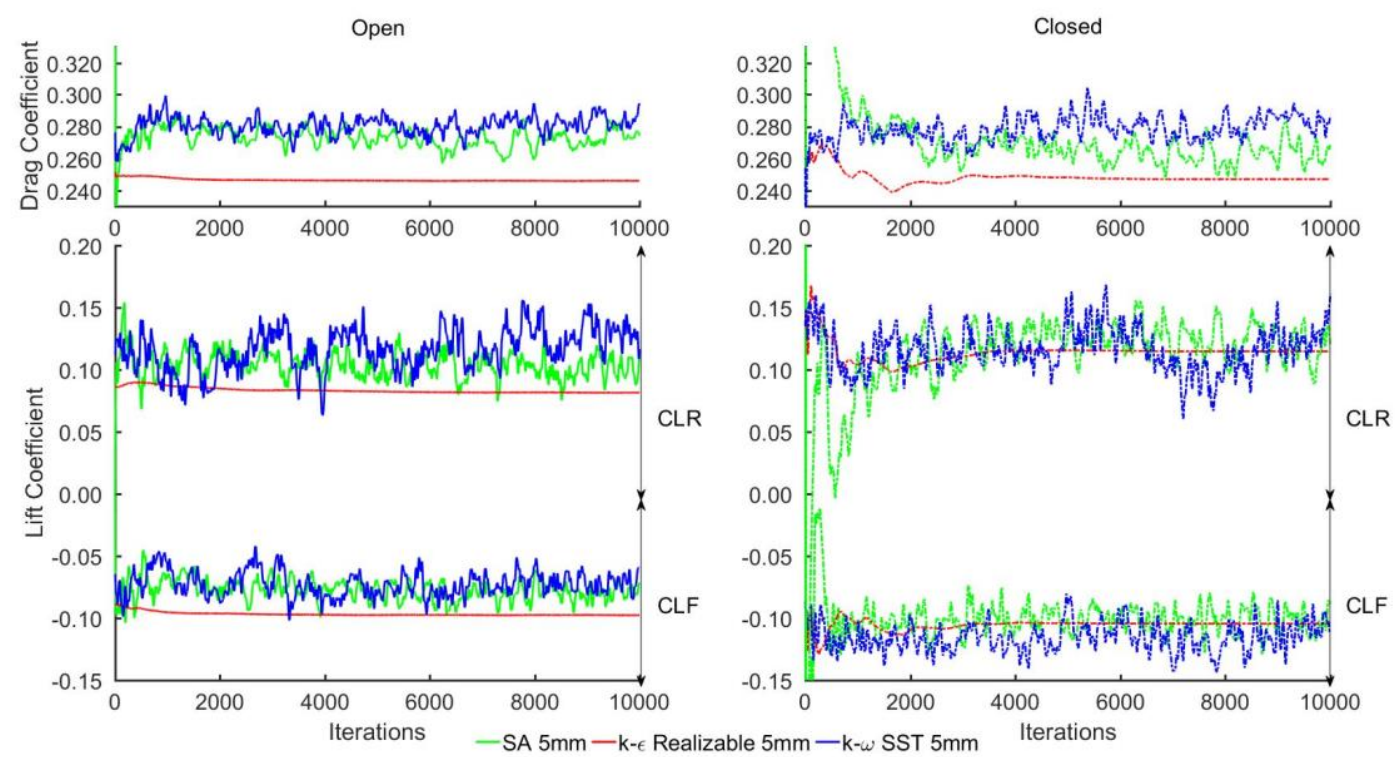

Figure 31. Lift and Drag residuals for the RANS and IDDES models in Closed configurations on the finest mesh 
2017-03-28

\section{Complete body aerodynamic study of three vehicles}

Simmonds, Nicholas

SAE International

Simmonds N, Pitman J, Tsoutsanis P, et al., (2017) Complete body aerodynamic study of three vehicles, Proceedings of WCX17: SAE World Congress Experience, 4 - 6 April 2017, Detroit, MI, Paper number 2017-01-1529

http://dx.doi.org/10.4271/2017-01-1529

Downloaded from Cranfield Library Services E-Repository 